TRANSACTIONS OF THE

AMERICAN MATHEMATICAL SOCIETY

Volume 356, Number 4 , Pages 1501-1533

S 0002-9947(03)03414-7

Article electronically published on September 22, 2003

\title{
STABILITY OF PARABOLIC HARNACK INEQUALITIES
}

\author{
MARTIN T. BARLOW AND RICHARD F. BASS
}

\begin{abstract}
Let $(G, E)$ be a graph with weights $\left\{a_{x y}\right\}$ for which a parabolic Harnack inequality holds with space-time scaling exponent $\beta \geq 2$. Suppose $\left\{a_{x y}^{\prime}\right\}$ is another set of weights that are comparable to $\left\{a_{x y}\right\}$. We prove that this parabolic Harnack inequality also holds for $(G, E)$ with the weights $\left\{a_{x y}^{\prime}\right\}$. We also give stable necessary and sufficient conditions for this parabolic Harnack inequality to hold.
\end{abstract}

\section{INTRODUCTION}

Consider the elliptic operator in divergence form

$$
\mathcal{A} f(x)=\sum_{i, j=1}^{d} \frac{\partial}{\partial x_{i}}\left(a_{i j}(\cdot) \frac{\partial f}{\partial x_{j}}\right)(x)
$$

acting on functions on $\mathbb{R}^{d}$, where $a=\left(a_{i j}(x)\right)$ is bounded, measurable, and uniformly elliptic. A celebrated theorem of Moser [M1] states that an elliptic Harnack inequality (EHI) holds for nonnegative functions $h$ that are harmonic with respect to the operator $\mathcal{A}$. This was extended a few years later in [M2], where Moser proved a parabolic Harnack inequality (PHI) for nonnegative solutions to the heat equation associated with $\mathcal{A}$ :

$$
\frac{\partial u}{\partial t}=\mathcal{A} u
$$

(See also M3.) These theorems have had a profound influence on linear and nonlinear PDE and differential geometry. To mention just one important result, the EHI lies behind Aronson's proof [A] of Gaussian type bounds for the fundamental solution to (1.2).

We can view Moser's theorems as stability theorems for elliptic and parabolic Harnack inequalities. It is well known that associated to the operator $\mathcal{A}$ is the Dirichlet form

$$
\mathcal{E}_{\mathcal{A}}(f, f)=\int_{\mathbb{R}^{d}} \sum_{i, j=1}^{d} a_{i j}(x) \frac{\partial f}{\partial x_{i}}(x) \frac{\partial f}{\partial x_{j}}(x) d x .
$$

Received by the editors January 24, 2003.

2000 Mathematics Subject Classification. Primary 60J27; Secondary 60J35, 31B05.

Key words and phrases. Harnack inequality, random walks on graphs, volume doubling, Green functions, Poincaré inequality, Sobolev inequality, anomalous diffusion.

The first author's research was partially supported by an NSERC (Canada) grant, and by CNRS (France).

The second author's research was partially supported by NSF Grant DMS 9988486. 
Of course, the Dirichlet form associated with the Laplacian is

$$
\mathcal{E}_{\Delta}(f, f)=\int_{\mathbb{R}^{d}}|\nabla f(x)|^{2} d x
$$

and the uniform ellipticity of $\mathcal{A}$ implies that the Dirichlet forms $\mathcal{E}_{\Delta}$ and $\mathcal{E}_{\mathcal{A}}$ are comparable in the sense that there exists $c_{1}>1$ such that

$$
c_{1}^{-1} \mathcal{E}_{\Delta}(f, f) \leq \mathcal{E}_{\mathcal{A}}(f, f) \leq c_{1} \mathcal{E}_{\Delta}(f, f), \quad f \in \mathbb{C}_{0}^{1}\left(\mathbb{R}^{d}\right) .
$$

Then we can rephrase Moser's theorems as:

Suppose the state space is $\mathbb{R}^{d}$. Whenever one has a Dirichlet form that is comparable to that of the Laplacian, then the EHI and PHI hold for the corresponding elliptic operator.

Moser's methods, and an alternate approach due to Fabes-Stroock [FS], building on work of Nash [N] and Davies [Da], have been used successfully in a variety of contexts: certain manifolds, domains in $\mathbb{R}^{d}$, graphs, and metric spaces. In particular, $\mathrm{Gr}$ and $[\mathrm{SC}]$ gave stable necessary and sufficient conditions for PHI to hold, by proving that the PHI with space-time scaling exponent 2, denoted $\mathrm{PHI}(2)$, holds on a manifold $M$ if and only if $M$ satisfies a volume doubling condition and a Poincaré inequality with scaling exponent 2. See [D1] and [St] for extensions of this to certain graphs and metric spaces.

No equivalent characterization for the EHI is known, and it was for some time an open problem whether the $\mathrm{EHI}$ and $\mathrm{PHI}(2)$ were equivalent. The (negative) answer was actually implicit in a number of papers on diffusions on fractals, before being made explicit in [BB1]. To explain the difference between these two Harnack inequalities further, consider the Markov process associated with the operator $\mathcal{A}$. PHI(2) implies that $X$ satisfies the standard space-time scaling relation:

$$
\mathbb{E}^{x}\left|X_{t}-x\right|^{2} \approx t
$$

In the early 1980s mathematical physicists discovered "fractal" lattices for which anomalous diffusion occurs:

$$
\mathbb{E}^{x} d\left(X_{n}, x\right)^{2} \approx n^{2 / \beta}, \quad n \geq 1,
$$

for some $\beta>2$. This property does not destroy the EHI, since the space-time scaling does not arise in the Laplace equation $\mathcal{A} h=0$, but it does affect the PHI, where the space-time scaling occurs explicitly. Thus these spaces do not satisfy $\operatorname{PHI}(2)$, but in many cases they do satisfy the EHI; see, for example, the graphs studied in [.J0 and [BB2]. The proofs of the EHI in these contexts generally employ some special features of the state space such as finite ramification or very strong symmetry conditions and do not give stability of the EHI.

The techniques of $[\mathrm{Gr}$ and $[\mathrm{SC}]$ do not apply to these spaces. The chief obstacle is that the Moser argument needs the existence of sufficiently many cut-off functions $\varphi$, with approximately minimal energy, such that the $L^{2}$ and $L^{\infty}$ norms of $\nabla \varphi$, suitably normalized, are comparable. Functions of this kind exist only if $\beta=2$.

Thus there exists a large family of spaces (see for example [B1] and [BCG]), corresponding to $\beta \in(2, \infty)$, for which EHI holds but PHI(2) fails. A parabolic Harnack inequality does hold for these spaces, but the space-time scaling exponent will be $\beta$ rather than 2 ; we denote this by $\operatorname{PHI}(\beta)$. In this paper we prove stability results for $\mathrm{PHI}(\beta)$ (Theorem 1.1) and give stable necessary and sufficient conditions for $\operatorname{PHI}(\beta)$ to hold (Theorem 1.5). These conditions include the obvious extensions 
of the $\beta=2$ case, that is, volume doubling and a rescaled Poincaré inequality, $\operatorname{PI}(\beta)$, but also a new inequality $\operatorname{CS}(\beta)$, which implies the existence of enough 'low energy' cut-off functions on the space.

The general question of the stability of EHI remains open. An example in [D2] shows that EHI can hold without a volume doubling property and also that, essentially, there exist spaces satisfying EHI with different $\beta$ in different regions. For other recent work on the relation between the EHI and PHI see [HSC].

We now introduce some notation and terminology to describe our results. It is generally the case that techniques for estimating heat kernels are quite robust and can be adapted to a variety of different spaces: graphs, manifolds, subsets of $\mathbb{R}^{d}$, fractals, and general metric spaces. We have chosen to work on graphs, since this provides the simplest context to employ our methods. However, we expect that our methods will transfer with only fairly minor changes to these other spaces.

We use the letter $c$ with subscripts to denote finite positive constants which depend only on the graph and whose exact value is unimportant. We use the notation $A \approx B$ to mean $c_{1} A \leq B \leq c_{2} A$, where $c_{1}$ and $c_{2}$ are as above.

Let $(G, E)$ be an infinite connected graph; $G$ is the set of vertices and $E$ is the set of edges. We suppose throughout that each vertex belongs to at most finitely many edges. We write $x \sim y$ to mean that $\{x, y\}$ is an edge. We call $a=\left(a_{x y}\right)$, $x, y \in G$, a conductance matrix if $a_{x y} \geq 0, a_{x y}=a_{y x}$ for all $x, y \in G$ and $a_{x y}=0$ if $\{x, y\}$ is not an edge in $E$. We call $(G, E, a)$ a weighted graph.

We set $\mu_{0}(\{x\})=\sum_{y} a_{x y}$ and we extend $\mu_{0}$ to a measure on $G$ by setting $\mu_{0}(A)=\sum_{x \in A} \mu_{0}(\{x\})$. Let $d(x, y)$ be the usual graph distance on $G$, and for $x \in G, r \in[0, \infty)$, set

$$
B(x, r)=\{y: d(x, y)<r\}, \quad V_{0}(x, r)=\mu_{0}(B(x, r)) .
$$

Given a ball $B=B(x, r)$, we write $B^{*}=B(x, 2 r)$.

We will introduce a number of conditions that a graph may or may not satisfy. For the convenience of the reader we give a table which summarizes what the abbreviations mean and where the definitions may be found.

$\begin{array}{lll}(\mathrm{VD}) & \text { Volume doubling } & (1.4) \\ \left(p_{0}\right) & \text { Lower bound on 1-step transition probabilities } & (1.5) \\ \left(E_{\beta}\right) & \text { Walk dimension } \beta & (1.6) \\ (\mathrm{EHI}) & \text { Elliptic Harnack inequality } & (1.8) \\ (\mathrm{PHI}(\beta)) & \text { Parabolic Harnack inequality with exponent } \beta & (1.9) \\ \left(R_{\beta}\right) & \text { Resistance exponent } \beta & (1.11) \\ (P I(\beta)) & \text { Poincaré inequality with exponent } \beta & (1.14) \\ (\mathrm{CS}(\beta)) & \text { Cut-off Sobolev inequality } & (1.15) \\ (\mathrm{FVG}) & \text { Fast volume growth } & (2.2)\end{array}$

The weighted graph $(G, E, a)$ satisfies the volume doubling condition (VD) if there exists $c_{1}>1$ such that

$$
V_{0}(x, 2 R) \leq c_{1} V_{0}(x, R) \quad \text { for all } x \in G, R \geq 1 .
$$

The simple random walk $X=\left(X_{n}, n \geq 0, \mathbb{P}^{x}, x \in G\right)$ on $(G, E, a)$ is the $\mu_{0^{-}}$ symmetric $G$-valued Markov chain with transition probabilities given by

$$
p_{x y}=\mathbb{P}^{\cdot}\left(X_{n+1}=y \mid X_{n}=x\right)=\frac{a_{x y}}{\mu_{0}(\{x\})}, \quad x, y \in G, n \geq 0 .
$$


We need a regularity condition which connects the $p_{x y}$ with the graph structure. We say that $(G, E, a)$ satisfies the $\left(p_{0}\right)$ condition if there exists $p_{0}>0$ such that

$$
p_{x y}=\frac{a_{x y}}{\mu_{0}(\{x\})} \geq p_{0} \quad \text { whenever }\{x, y\} \text { is an edge in } E .
$$

Note that this implies that vertices have degree at most $p_{0}^{-1}$.

The heat kernel on $(G, E, a)$ is the density of $X_{n}$ with respect to the measure $\mu_{0}$ :

$$
p_{n}(x, y)=\frac{\mathbb{P}^{x}\left(X_{n}=y\right)}{\mu_{0}(\{y\})},
$$

and is easily seen to be symmetric: $p_{n}(x, y)=p_{n}(y, x)$. Here we use the Markov theory notation $\mathbb{P}^{x}(\cdot)=\mathbb{P}\left(\cdot \mid X_{0}=x\right)$ and denote the corresponding expectation operator by $\mathbb{E}^{x}$. We say that $(G, E, a)$ has walk dimension $\beta$ and that $(G, E, a)$ satisfies $\left(E_{\beta}\right)$ if for some constant $c_{1} \geq 1$,

$$
c_{1}^{-1} r^{\beta} \leq \mathbb{E}^{x} \tau_{B(x, r)}^{X} \leq c_{1} r^{\beta}, \quad r \in[1, \infty), x \in G,
$$

where $\tau_{B(x, r)}^{X}=\min \left\{n: X_{n} \notin B(x, r)\right\}$. The term walk dimension comes from the mathematical physics literature; cf. [BB1], Proposition 8.3.

We next define (EHI), the elliptic Harnack inequality. The graph Laplacian $\mathcal{L}_{G}$ is defined by

$$
\mathcal{L}_{G} f(x)=\frac{1}{\mu_{0}(\{x\})} \sum_{y} a_{x y}(f(y)-f(x)), \quad x \in G .
$$

Note that $\mathcal{L}_{G}$ depends on the conductance matrix $a$. A function $h: \bar{A} \rightarrow \mathbb{R}$ is harmonic on $A \subset G$ if

$$
\mathcal{L}_{G} h(x)=0, \quad x \in A,
$$

where $\bar{A}=\{y \in G: d(x, y) \leq 1$ for some $x \in A\}$. $(G, E, a)$ satisfies an elliptic Harnack inequality (EHI) if there exists $c_{1}>0$ such that: whenever $x \in G, R \geq 1$, and $h: G \rightarrow \mathbb{R}$ is nonnegative and harmonic in $B(x, 2 R)$,

$$
\sup _{B(x, R)} h \leq c_{1} \inf _{B(x, R)} h .
$$

We have taken balls $B(x, R) \subset B(x, 2 R)$ just for simplicity: if $K>1$ and (1.8) holds whenever $h \geq 0$ is harmonic in $B(x, K R)$, then an easy chaining argument gives (EHI) (for a different constant $c_{1}$ ). We remark that under the $\left(p_{0}\right)$ condition any graph satisfies a local Harnack inequality; see Lemma 2.8. The point of the condition (EHI) is that the constant $c_{1}$ is independent of $x$ and $R$.

Let $\beta \geq 2$. $(G, E, a)$ satisfies $\operatorname{PHI}(\beta)$, a parabolic Harnack inequality of order $\beta$, if whenever $u(n, x) \geq 0$ is defined on $[0,4 N] \times \bar{B}(y, 2 R)$ and satisfies

$$
u(n+1, x)-u(n, x)=\mathcal{L}_{G} u(n, x), \quad(n, x) \in[0,4 N) \times B(y, 2 R),
$$

then

$$
\max _{\substack{N \leq n \leq 2 N \\ x \in B(y, R)}} u(n, x) \leq c_{1} \min _{\substack{3 N \leq n \leq 4 N \\ x \in B(y, R)}}(u(n, x)+u(n+1, x))
$$

where $N \geq 2 R$ and $N \approx R^{\beta}$. The usual parabolic Harnack inequality is the case $\beta=2$; this extension was introduced in [BB1].

Suppose that $a$ and $a^{\prime}$ are conductance matrices on $(G, E)$. We say $a$ and $a^{\prime}$ are equivalent if there exists a constant $c_{1}$ such that

$$
c_{1}^{-1} a_{x y} \leq a_{x y}^{\prime} \leq c_{1} a_{x y}, \quad\{x, y\} \in E .
$$


We call a property stable if whenever it holds for $(G, E, a)$ and $a$ and $a^{\prime}$ are equivalent, then it holds for $\left(G, E, a^{\prime}\right)$. Our first main result is that $(\operatorname{PHI}(\beta))$ is stable.

Theorem 1.1. Suppose that $(G, E)$ is an infinite connected graph and that $a$ and $a^{\prime}$ are equivalent conductance matrices on $(G, E)$ satisfying the $\left(p_{0}\right)$ condition. Suppose that the weighted graph $(G, E, a)$ satisfies $P H I(\beta)$. Then $\left(G, E, a^{\prime}\right)$ also satisfies $\operatorname{PHI}(\beta)$.

If $A$ and $B$ are two disjoint subsets of $G$, define the effective resistance between $A$ and $B$ by

$$
\left[R_{\mathrm{eff}}(A, B)\right]^{-1}=\inf \left\{\sum_{x \sim y}|f(x)-f(y)|^{2} a_{x y}: f=1 \text { on } A, f=0 \text { on } B\right\} .
$$

We say $G$ satisfies $\left(R_{\beta}\right)$ and has resistance exponent $\beta$ if

$$
R_{\mathrm{eff}}\left(B\left(x_{0}, r\right), B\left(x_{0}, 2 r\right)^{c}\right) \approx \frac{r^{\beta}}{V_{0}\left(x_{0}, r\right)}, \quad r \geq 1 .
$$

Note that the property $\left(R_{\beta}\right)$ is stable.

Many properties of random walks on graphs satisfying $\operatorname{PHI}(\beta)$ are already quite well known; in particular one has good estimates on the transition probabilities $p_{n}(x, y)$. The following is the main theorem of [GT2].

Theorem 1.2 (GT2, Theorem 3.1]). Let $(G, E)$ be an infinite connected graph with conductances $a=\left(a_{x y}\right)$ satisfying the $\left(p_{0}\right)$ condition. The following are equivalent:

(a) $(G, E, a)$ satisfies $P H I(\beta)$.

(b) $(G, E, a)$ satisfies $(V D),\left(E_{\beta}\right)$, and (EHI).

(c) For $x, y \in G, n \geq d(x, y)$, the transition density of $X$ on $(G, E, a)$ satisfies

$$
\begin{aligned}
p_{n}(x, y) & \leq \frac{c_{1}}{V_{0}\left(x, n^{1 / \beta}\right)} \exp \left[-\left(\frac{d(x, y)^{\beta}}{c_{1} n}\right)^{1 /(\beta-1)}\right], \\
p_{n}(x, y)+p_{n+1}(x, y) & \geq \frac{c_{2}}{V_{0}\left(x, n^{1 / \beta}\right)} \exp \left[-\left(\frac{d(x, y)^{\beta}}{c_{2} n}\right)^{1 /(\beta-1)}\right] .
\end{aligned}
$$

(d) $(G, E, a)$ satisfies (VD), (EHI) and $\left(R_{\beta}\right)$.

Remarks 1.3. 1. We always have $\beta \geq 2$; see, for example, [B1], Lemma 1.1 - the proof extends easily to the volume doubling case.

2. Explicit examples of graphs satisfying these conditions, for various $\beta \geq 2$, are given in [B1], [BB2], and [Jo].

3. Note that $p_{n}(x, y)=0$ when $d(x, y)>n$. The purpose of adding $p_{n}$ and $p_{n+1}$ in (1.13) is to avoid the parity problems which arise if $G$ is bipartite or close to bipartite.

We prove Theorem 1.1 by finding stable necessary and sufficient conditions for $\operatorname{PHI}(\beta)$ to hold for a graph which satisfies $\left(p_{0}\right)$.

$(G, E, a)$ satisfies $(P I(\beta))$, a scaled Poincaré inequality with parameter $\beta \geq 2$, if there exists a constant $c_{1}$ such that for any ball $B=B(x, R) \subset G$ with $R \geq 1$, and $f: B \rightarrow \mathbb{R}$,

$$
\sum_{x \in B}\left(f(x)-\bar{f}_{B}\right)^{2} \mu_{0}(x) \leq c_{1} R^{\beta} \sum_{x \in B} \sum_{y \in B} a_{x y}(f(x)-f(y))^{2} .
$$

Here $\bar{f}_{B}=\mu(B)^{-1} \sum_{B} f(x) \mu_{0}(x)$. This is a generalization to the anomalous diffusion case of the standard Poincaré inequality. 
The following definition is new.

Definition 1.4. $\operatorname{CS}(\beta)$, the cut-off Sobolev inequality with exponents $\beta$ and $\theta$.

Let $\beta \geq 2$ and $\theta \in(0,1]$. We say $(G, E, a)$ satisfies $\operatorname{CS}(\beta)$ if there exist constants $c_{1}$ and $c_{2}$ such that for every $x_{0} \in G, R \geq 1$, there exists a cut-off function $\varphi\left(=\varphi_{x_{0}, R}\right)$ satisfying properties (a)-(d) below.

(a) $\varphi(x) \geq 1$ for $x \in B\left(x_{0}, R / 2\right)$.

(b) $\varphi(x)=0$ for $x \in B\left(x_{0}, R\right)^{c}$.

(c) $|\varphi(x)-\varphi(y)| \leq c_{1}(d(x, y) / R)^{\theta}$ for all $x, y$.

(d) For any ball $B\left(x_{1}, s\right)$ with $1 \leq s \leq R$ and $f: B\left(x_{1}, 2 s\right) \rightarrow \mathbb{R}$

$$
\begin{aligned}
& \sum_{x \in B\left(x_{1}, s\right)} f(x)^{2} \sum_{y \in G} a_{x y}|\varphi(x)-\varphi(y)|^{2} \\
& \leq c_{2}\left(\frac{s}{R}\right)^{2 \theta}\left(\sum_{x \in B\left(x_{1}, 2 s\right)} \sum_{y \in B\left(x_{1}, 2 s\right)} a_{x y}|f(x)-f(y)|^{2}+s^{-\beta} \sum_{x \in B\left(x_{1}, 2 s\right)} \mu_{0}(x) f(x)^{2}\right) .
\end{aligned}
$$

We also call (1.15) a weighted Sobolev inequality relative to $\varphi$, with exponent $\beta$ and scale $R$.

We can now state our second main theorem.

Theorem 1.5. Suppose that $(G, E, a)$ is an infinite connected weighted graph satisfying the $\left(p_{0}\right)$ condition. The following are equivalent:

(a) There exists $\theta \in(0,1]$ such that $(G, E, a)$ satisfies (VD), PI( $\beta)$ and $C S(\beta)$.

(b) $(G, E, a)$ satisfies $P H I(\beta)$.

Remarks 1.6. 1. It is obvious that the conditions $P I(\beta)$ and $\operatorname{CS}(\beta)$ are stable, so that Theorem 1.1 is an immediate consequence of Theorem 1.5.

2. Let $2 \leq \beta \leq \beta^{\prime}$. Then it is easy to see that $P I(\beta)$ implies $P I\left(\beta^{\prime}\right)$, while $\operatorname{CS}\left(\beta^{\prime}\right)$ implies $\operatorname{CS}(\beta)$. In fact it is easy to check that $\operatorname{CS}(2)$ always holds; essentially one can take $\varphi(x)=(2 / R) d\left(x, B\left(x_{0}, R\right)^{c}\right)$. Thus $|\varphi(x)-\varphi(y)| \leq 2 / R$ if $a_{x y}>0$, and (1.15) follows easily. In view of this we can regard Theorem 1.5 as an extension of the characterization of $\mathrm{PHI}(2)$ due to $[\mathrm{Gr}]$ and $[\mathrm{SC}]$ to the $\beta>2$ case: the extra hypothesis $\operatorname{CS}(2)$ is always true.

3. It is easy to prove (see Lemma 5.1) that if $P I(\beta)$ holds, then $\operatorname{CS}\left(\beta^{\prime}\right)$ cannot hold for any $\beta^{\prime}>\beta$.

4. If $\left(G_{i}, E_{i}, a_{i}\right), i=1,2$, are two graphs satisfying $\operatorname{PHI}\left(\beta_{i}\right)$, respectively, with $\beta_{1}<\beta_{2}$, then the product $G=G_{1} \times G_{2}$ satisfies $P I\left(\beta_{2}\right)$. However, since $G$ does not satisfy $\operatorname{PHI}\left(\beta_{2}\right)$ it cannot satisfy $\operatorname{CS}\left(\beta_{2}\right)$ for any $\theta$. Thus the conditions $P I(\beta)$ and $\operatorname{CS}(\beta)$ are independent.

5 . We give the corresponding version of $\operatorname{CS}(\beta)$ for a non-discrete space below see Definition 3.1.

6. It is likely that the results of this paper can be extended to more general time-scale functions $F$ than the case $F(R)=R^{\beta}$ considered here. For some recent work involving these more general types of space-time scaling, see [HSC] and [T2].

7. The conditions (VD), $P I(\beta)$ and $\mathrm{CS}(\beta)$ are stable under rough isometries see [HK], Proposition 5.15.

We now give a brief sketch of the proof that (a) implies (b) in Theorem 1.5. Given a harmonic function $u \geq 0$, and writing $f=u^{p}$, Moser's proof uses a Sobolev inequality to bound $\int_{Q}|f|^{2+\varepsilon}$ in terms of $\int_{Q}|\nabla f|^{2}$. This integral of $\nabla f$ is then 
bounded in terms of $\int_{Q^{\prime}}|f|^{2}$ : here $Q \subset Q^{\prime}$ are two cubes of slightly different sizes. Iterating and passing to the limit, one obtains a bound on the $L^{\infty}$ norm of $u$. One performs a similar argument for negative powers, and then one links the positive and negative powers.

For spaces with $\beta>2$ difficulties arise with the initial iteration argument. In order to bound $\int|\nabla f|^{2}$ in terms of $\int|f|^{2}$, one needs to perform an integration by parts, and it is here that one needs suitable cut-off functions. Spaces with $\beta>2$ typically have a large number of holes, and this means that the minimum energy cut-off function $\varphi$ such that $\varphi=1$ on a ball $B(x, R / 2)$, and $\varphi=0$ outside $B(x, R)$, will satisfy

$$
\int_{B(x, R)}|\nabla \varphi|^{2} d \mu_{0} \approx R^{-\beta} V_{0}(x, R) \ll R^{-2} V_{0}(x, R) .
$$

(One can use the 'bottlenecks' in the space to find functions with substantially lower energy than the obvious linear function.) The standard Moser iteration argument (and also the method of Davies) uses cut-off functions $\varphi$ with $\|\nabla \varphi\|_{\infty} \approx R^{-2}$, so that one can bound $\int_{B(x, R)} f^{2}|\nabla \varphi|^{2}$ by $R^{-2} \int_{B(x, R)} f^{2}$. These bounds still hold in the anomalous diffusion case, but are not enough: one needs $R^{-\beta}$ rather than $R^{-2}$ to cancel terms involving $R^{\beta}$ which arise from the Poincaré inequality $P I(\beta)$. Thus for spaces with $\beta>2$, cut-off functions for which one has good enough control of $\|\nabla \varphi\|_{\infty}$ do not exist. (This is related to the fact, proved in [K] in some special cases, that in the scaling limit, the energy measure given formally by $|\nabla f|^{2} d \mu_{0}$ is in fact singular with respect to $\mu_{0}$.)

What we do instead is use $\operatorname{CS}(\beta)$ to find a cut-off function $\varphi$ and prove that

$$
\int_{B\left(x_{0}, R / 2\right)}|\nabla f|^{2} \leq c_{1} \int_{B\left(x_{0}, R\right)}|f|^{2} d \nu
$$

for the measure $\nu=\left(1+R^{\beta}|\nabla \varphi|^{2}\right) d \mu_{0}$. This measure $\nu$ is not very tractable in general - we do not, for example, know if it satisfies volume doubling. However, we can prove a weighted Sobolev inequality linking the $L^{2+\varepsilon}$ norm of $f$ with respect to $\nu$ to the $L^{2}$ norm of $\nabla f$ with respect to $\mu_{0}$.

In BB3] we used a version of this argument to prove Harnack inequalities for certain fractal-like domains in $\mathbb{R}^{d}$. However, the symmetry available in that context played an essential role at several points. In the current paper no symmetry is assumed. Some other differences, although less crucial, are (i) we allow the weaker condition of volume doubling rather than the condition $V(x, R) \approx R^{\alpha}$ that was present in [BB3, and (ii) we need to circumvent the potentially bad decay of harmonic functions near the boundaries of balls.

In working with graphs one encounters numerous minor difficulties arising from the discrete structure. One possibility would be to deal with these directly, as is done for example in [D1]. However, as in this paper we are concerned with harmonic functions, we can embed the graph $G$ in a connected metric space (called the cable system for the graph) and prove the Harnack inequality in that context. It is then easy to obtain the result for the original graph.

In Section 2 we recall the cable system associated with the graph $(G, E, a)$, and derive some needed properties. In Section 3 we prove the equivalence of $\operatorname{CS}(\beta)$ on the graph $G$ and cable system $G_{C}$. We prove that implication (b) implies (a) in Theorem 1.5 in Section 4 by using properties of Green functions to build a suitable 
cutoff function $\varphi$. In Section 5 we use the Moser iteration argument outlined above to prove that (a) implies (b).

\section{THE CABLE SYSTEM AND PRELIMINARIES}

We note that the condition (VD) implies that there exists $\alpha_{2}>0$ such that if $x$, $y \in G$ and $0<r<R$, then

$$
\frac{V_{0}(x, R)}{V_{0}(y, r)} \leq c_{1}\left(\frac{d(x, y)+R}{r}\right)^{\alpha_{2}} .
$$

We introduce the following fast volume growth (FVG) condition: there exists $\alpha_{1}>0$ such that if $x, y \in G$ and $0<r<R$, then

$$
\frac{V_{0}(x, R)}{V_{0}(x, r)} \geq c_{2}\left(\frac{R}{r}\right)^{\alpha_{1}} .
$$

Observe that if both (2.1) and (2.2) hold, then $\alpha_{1} \leq \alpha_{2}$. Given $\operatorname{PHI}(\beta)$, the condition (FVG) (with $\alpha_{1}>\beta$ ) implies that the Markov chain $X$ is transient and gives good control over the decay of the Green functions.

We now introduce the cable system associated with $(G, E, a)$. Loosely speaking, this is the metric space $G_{C}$ obtained by replacing each edge in $E$ by a 'cable' of length 1 . The associated Markov process behaves like a linear Brownian motion on each cable and when at a vertex picks the edge on which the next excursion will lie according to the conductances $a_{x y}$. (These processes are sometimes called 'Walsh Brownian motions' - see [W], [BPY].)

To be more precise, we let $G_{C}$ consist of $G$ together with cables, one for each edge. Each cable is a copy of $(0,1)$. We let $\mu(d x)=a_{x y} d x$ on the cable $C(x, y)$ associated with the edge $\{x, y\} ; \mu$ assigns no mass to any vertex. The distance between two points $x$ and $y$ is given as follows: if $x$ and $y$ are on the same cable, the length is just the usual Euclidean distance $|x-y|$. If they are on different cables, then the distance is $\min \left\{\left|x-z_{x}\right|+d\left(z_{x}, z_{y}\right)+\left|z_{y}-y\right|\right\}$, where the minimum is taken over all vertices $z_{x}$ and $z_{y}$ such that $x$ is on a cable with one end at $z_{x}$ and $y$ is on a cable with one end at $z_{y}$. We denote this distance by $d(x, y)$ as well, since it generalizes the graph distance. We again let $B(x, r)=\left\{y \in G_{C}: d(x, y)<r\right\}$ denote the ball of radius $r$ and set $V(x, r)=\mu(B(x, r))$.

The following lemma is easy to check.

Lemma 2.1. The following are equivalent:

(a) $(G, E, a)$ satisfies $\left(p_{0}\right)$ and $(V D)$ with respect to $\mu_{0}$.

(b) $G_{C}$ satisfies (VD) with respect to $\mu$. Moreover, if $R \geq 1$,

$$
V_{0}(x, R) \approx V(x, R) .
$$

Given a function $f$ on $G_{C}$ and a point $x$ in the interior of a cable, we define $\nabla f(x)$ as follows. We choose an orientation for the edges of $G$, and if $x$ is in the oriented edge $\left\{y_{0}, y_{1}\right\}$, then we set

$$
\nabla f(x)=\lim _{z \rightarrow x} \frac{f(z)-f(x)}{d\left(y_{0}, z\right)-d\left(y_{0}, x\right)} .
$$

If $x \in G$, and $\{x, y\}$ is an edge, write $C(x, y)$ for the cable containing $x$ and $y$ and let

$$
\nabla_{y} f(x)=\lim _{z \rightarrow x, z \in C(x, y)} \frac{f(z)-f(x)}{d(x, z)} .
$$


Note that while the choice of orientation will affect the sign of $\nabla f$, it will not affect the quantities $\nabla_{y} f,|\nabla f(z)|^{2}, \nabla f(z) \nabla g(z)$, or $\nabla^{2} f$. We then let

$$
\mathcal{E}_{Y}(f, f)=\frac{1}{2} \sum_{\{x, y\} \in E} \int|\nabla f(z)|^{2} \mu(d z),
$$

and let $\mathcal{D}_{0}\left(\mathcal{E}_{Y}\right)$ be the collection of continuous functions from $G_{C}$ to $\mathbb{R}$ with compact support such that $\nabla f(y)$ exists at every point of $G_{C}-G, \nabla_{y} f(x)$ exists at every point $x \in G$ and for every cable $C(x, y)$, and $\|\nabla f\|_{\infty}<\infty$. Let $\mathcal{D}\left(\mathcal{E}_{Y}\right)$, the domain of $\mathcal{E}_{Y}$, be the completion of $\mathcal{D}_{0}\left(\mathcal{E}_{Y}\right)$ with respect to the norm

$$
\left(\sum_{\{x, y \in E\}} \int|f(z)|^{2} \mu(d z)\right)^{1 / 2}+\left(\mathcal{E}_{Y}(f, f)\right)^{1 / 2} .
$$

The cable process is the symmetric continuous Markov process $Y$ which corresponds to the Dirichlet form $\left(\mathcal{E}_{Y}, \mathcal{D}\left(\mathcal{E}_{Y}\right)\right)$ on $L^{2}\left(G_{C}, \mu\right)$ - see [FOT]. We denote the infinitesimal generator of $Y$ by $\mathcal{L}$ and its domain by $\mathcal{D}(\mathcal{L})$. If $x \in G_{C}-G$, then we have $\mathcal{L} f(x)=\frac{1}{2} \nabla^{2} f(x)$. To describe $\mathcal{L} f$ at points $x \in G$, we need some additional definitions.

Definition 2.2. Let $D$ be an open domain in $G_{C}$. Write $\mathcal{K}(D)$ for the set of functions $f: \bar{D} \rightarrow \mathbb{R}$ satisfying the following conditions.

(1) $f$ is continuous on $\bar{D}$.

(2) $\nabla f$ exists and is continuous on each cable in $D$, and $\lim _{x \rightarrow z} \nabla f(x)$ exists at each endpoint $z$ of a cable in $D$.

(3) There exists a discrete subset $\Gamma \subset D$ such that $\nabla^{2} f(x)$ exists and is continuous at all $x \in D-(G \cup \Gamma)$. If $I$ is an interval contained in a cable in $D$ and $z \in G \cup \Gamma$ is an endpoint of $I$, then $\lim _{x \rightarrow z, x \in I} \nabla^{2} f(x)$ exists.

(4) If $x \in G \cap D$, let $x_{i}, 1 \leq i \leq m$, be the $m$ neighbors of $x$ in $G$, and let $p_{i}=a_{x x_{i}} / \sum_{j=1}^{m} a_{x x_{j}}$. Then the directional gradients $\nabla_{x_{i}} f(x)$ satisfy the consistency condition

$$
\sum_{i=1}^{m} p_{i} \nabla_{x_{i}} f(x)=0 .
$$

We then have $\mathcal{K}\left(G_{C}\right) \subset \mathcal{D}(\mathcal{L})$, and for $f \in \mathcal{K}\left(G_{C}\right)$

$$
\mathcal{L} f(x)= \begin{cases}\frac{1}{2} \nabla^{2} f(x), & \text { if } x \in G_{C}-G, \\ \frac{1}{2} \sum_{i=1}^{m} p_{i} \nabla_{x_{i}}^{2} f(x) & \text { if } x \in G,\end{cases}
$$

where we write $\nabla_{x_{i}}^{2} f(x)=\lim _{z \rightarrow x, z \in C\left(x, x_{i}\right)} \nabla^{2} f(x)$.

For $D$ such that $\partial D \cap G=\emptyset$ and for $z \in \partial D$ we define the inward pointing normal derivative

$$
\frac{\partial f}{\partial n}(z)=\lim _{y \rightarrow z, y \in D} \frac{f(y)-f(z)}{d(y, z)} .
$$

Lemma 2.3 (Gauss-Green lemma). Let $D$ be an open subset of $G_{C}$ satisfying the condition that $\partial D \cap G=\emptyset$, and the intersection of $D$ with any cable consists of finitely many intervals. Let $f \in \mathcal{K}(D)$ and $g \in \mathcal{D}\left(\mathcal{E}_{Y}\right)$. Then

$$
\int_{D} g(z) \mathcal{L} f(z) d \mu(z)+\int_{D} \nabla g(z) \nabla f(z) d \mu(z)=-\sum_{\partial D} a_{e(z)} g(z) \frac{\partial f}{\partial n}(z) .
$$

Here e(z) denotes the cable containing $z$. 
Proof. Let $x \in D$ and $\{x, y\}$ be an edge in $E$. Then if $C(x, y) \subset D$, by standard integration by parts on $[0,1]$ we have

$$
\int_{C(x, y)}[g(z) \mathcal{L} f(z)+\nabla g(z) \nabla f(z)] a_{x y} d z=a_{x y}\left[g(y) \nabla_{x} f(y)-g(x) \nabla_{y} f(x)\right] .
$$

Similarly, if $C(x, y) \cap \partial D=\{z\}$, then

$$
\int_{C(x, y)}[g(z) \mathcal{L} f(z)+\nabla g(z) \nabla f(z)] a_{x y} d z=a_{x y}\left[g(y) \nabla_{x} f(y)-g(z) \frac{\partial f}{\partial n}(z)\right] .
$$

Summing these identities over all the cables which intersect $D$ and using (2.5), we obtain (2.8).

Let $B \subset G_{C}$ and $h: \bar{D} \rightarrow \mathbb{R}$. We say $h$ is harmonic (with respect to $\mathcal{L}$ ) if $h \in \mathcal{K}(D)$ and $\mathcal{L} h(x)=0$ for $x \in D$. The relation between $\mathcal{L}_{G}$ and $\mathcal{L}$ harmonic functions is given by the following lemma.

Lemma 2.4. (a) Let $A \subset G, h: \bar{A} \rightarrow \mathbb{R}$ and $h$ be $\mathcal{L}_{G}$-harmonic on $A$. Let $B$ be the open subset of $G_{C}$ consisting of $A$ and the interior of any cable with at least one endpoint in $A$. Define $\widetilde{h}$ by setting $\widetilde{h}(x)=h(x)$ for $x \in \bar{A}$, and extend $\widetilde{h}$ to a function on $\bar{B}$ by taking $\widetilde{h}$ to be linear on each cable. Then $\widetilde{h} \in \mathcal{K}(B)$ and $\widetilde{h}$ is $\mathcal{L}$-harmonic on $B$.

(b) Let $B \subset G_{C}$ and let $\widetilde{h}: \bar{B} \rightarrow \mathbb{R}$ be $\mathcal{L}$-harmonic. Set $A=\left\{x \in G: d\left(x, B^{c}\right)>\right.$ $1\}$. Then $\left.\widetilde{h}\right|_{A}$ is $\mathcal{L}_{G}$-harmonic.

Proof. (a) It is clear that $\widetilde{h}$ is continuous on $\bar{B}$ and that $\nabla^{2} \widetilde{h}=0$ on the interior of every cable. So $\widetilde{h}\left(x_{i}\right)=\widetilde{h}(x)+\nabla_{x_{i}} \widetilde{h}(x)$ for $1 \leq i \leq m$. Thus if $x \in G$,

$$
\mu_{0}(x)^{-1} \sum_{i} a_{x x_{i}} \nabla_{x_{i}} \widetilde{h}(x)=\mu_{0}(x)^{-1} \sum_{i} a_{x x_{i}}\left(h\left(x_{i}\right)-h(x)\right)=\mathcal{L}_{G} h(x)=0,
$$

so that $\widetilde{h}$ satisfies the consistency condition (2.5) at $x$. Since $\nabla_{x_{i}}^{2} h(x)=0$ for each $i$ if $x \in G$, we have $\mathcal{L} \widetilde{h}(x)=0$. Thus $\widetilde{h} \in \mathcal{K}(\bar{B})$, and $\widetilde{h}$ is $\mathcal{L}$-harmonic.

(b) is proved in a similar fashion.

Corollary 2.5. (EHI) holds for the $\mathcal{L}_{G}$-harmonic functions on the graph $G$ if and only if it holds for $\mathcal{L}$-harmonic functions on the cable system $G_{C}$.

Set $\tau_{B(x, r)}=\inf \left\{t: Y_{t} \notin B(x, r)\right\}$.

Lemma 2.6. If $(G, E, a)$ satisfies $\left(E_{\beta}\right)$, then $\mathbb{E}^{x} \tau_{B(x, r)} \approx r^{\beta}$ for $r \geq 1$.

Proof. Suppose first that $x \in G$ and $r$ is a positive integer. Let $S_{i}$ be the times that the cable process $Y_{t}$ hits successive vertices of $G$ and let $V_{i}=Y_{S_{i}}$. Writing $\xi_{i}=S_{i}-S_{i-1}, \xi_{i}$ is just the time for a standard one dimensional Brownian motion to move a distance 1 . So $\mathbb{E} \xi_{i}=1$ and the $\xi_{i}$ are i.i.d. random variables, which are independent of the process $V$. Since $V_{i}$ has the same distribution as the Markov chain $X_{i}$ we deduce, writing $\tau_{V}=\min \left\{n \geq 0: V_{n} \in B(x, r)^{c}\right\}$,

$$
\mathbb{E}^{x} \tau_{B(x, r)}=\mathbb{E}^{x} \sum_{i=1}^{\tau_{V}} \xi_{i}=\mathbb{E}^{x} \tau_{V} \approx r^{\beta} .
$$

The case of general $x$ and $r$ now follows easily. 
We will need the following covering lemma.

Lemma 2.7. Let $D \subset G_{C}$ have compact closure, and let $s>0$. There exists a cover of $D$ by balls $B\left(x_{i}, s\right)$ with $x_{i} \in D$ such that no point in $G_{C}$ is in more than $M$ of the $B\left(x_{i}, 2 s\right)$. Here $M$ depends only on $G_{C}$.

Proof. Let $x_{1} \in D$ and choose $x_{2}, x_{3}, \ldots$ by letting $x_{i+1}$ be any point in $D-$ $\bigcup_{j=1}^{i} B\left(x_{j}, s\right)$. We do this until we can no longer proceed. Note that the $x_{i}$ must be at least distance $s$ apart, so the balls $B\left(x_{i}, s / 2\right)$ are disjoint. Now suppose $y$ is in $m$ of the balls $B\left(x_{i}, 2 s\right)$. Then $B(y, 3 s)$ contains $m$ disjoint balls $B\left(x_{i}, s / 2\right)$, and using (2.1) we have for each of these

$$
\frac{V(y, 3 s)}{V\left(x_{i}, s / 2\right)} \leq c_{1}
$$

Thus

$$
V(y, 3 s) \geq \sum_{\left\{i: y \in B\left(x_{i}, 2 s\right)\right\}} V\left(x_{i}, s / 2\right) \geq m c_{1}^{-1} V(y, 3 s),
$$

and so we can take $M=c_{1}$.

We finally remark that under the $\left(p_{0}\right)$ condition any graph satisfies a local Harnack inequality.

Lemma 2.8. Suppose $A$ is a finite connected set. There exists $c_{1}$ depending only on $A$ such that if $h: \bar{A} \rightarrow \mathbb{R}$ is nonnegative and harmonic in $A$, then

$$
h(y) \leq c_{1} h(x), \quad x, y \in A .
$$

Proof. If $x_{i}, x_{i+1} \in A$ with $\left\{x_{i}, x_{i+1}\right\} \in E$, then

$$
h\left(x_{i}\right)=\sum_{z} p_{x_{i} z} h(z) \geq p_{x_{i} x_{i+1}} h\left(x_{i+1}\right) \geq p_{0} h\left(x_{i+1}\right) .
$$

Since $A$ is connected, if $x, y \in A$, we can find $x=x_{0}, x_{1}, \ldots, x_{n-1}, x_{n}=y$ such that each $x_{i} \in A$ and each $\left\{x_{i}, x_{i+1}\right\} \in E$. Since $A$ is finite, we can take $n$ less than or equal to the cardinality of $A$. By using (2.9) at most $n-1$ times, we obtain our result.

\section{The Cut-off Sobolev inequality}

We give the form of the cut-off Sobolev inequality for the cable system. Define the function

$$
\psi(r)= \begin{cases}r^{\beta}, & \text { if } r \geq 1, \\ r^{2}, & \text { if } r \leq 1 .\end{cases}
$$

Note that we can also write $\psi(r)=r^{\beta} \vee r^{2}$ and that $\mathbb{E}^{x} \tau_{B(x, r)} \approx \psi(r)$.

Definition 3.1. $G_{C}$ satisfies $\operatorname{CS}(\beta)$ for $\beta \geq 2$ and $\theta \in(0,1]$ if there exist constants $c_{1}$ and $c_{2}$ such that the following holds. For every $x_{0} \in G_{C}, R>0$ there exists a function $\varphi\left(=\varphi_{x_{0}, R}\right)$ with the following properties:

(a) $\varphi(x) \geq 1$ for $x \in B\left(x_{0}, R / 2\right)$.

(b) $\varphi(x)=0$ for $x \in B\left(x_{0}, R\right)^{c}$.

(c) $|\varphi(x)-\varphi(y)| \leq c_{1}(d(x, y) / R)^{\theta}$ for all $x, y$.

(d) For any ball $B(x, s)$ with $0<s \leq R$ and $f: B(x, 2 s) \rightarrow \mathbb{R}$

$$
\int_{B(x, s)} f^{2}|\nabla \varphi|^{2} d \mu \leq c_{2}(s / R)^{2 \theta}\left(\int_{B(x, 2 s)}|\nabla f|^{2} d \mu+\psi(s)^{-1} \int_{B(x, 2 s)} f^{2} d \mu\right) .
$$


We now compare $\operatorname{CS}(\beta)$ for the graph $G$ and cable system $G_{C}$. We begin with some elementary remarks.

Remarks 3.2. 1. Suppose $\operatorname{CS}(\beta)$ holds for $G_{C}$, but with (a) above replaced by

$$
\varphi(x) \geq 1 \text { for } x \in B\left(x_{0}, \delta R\right),
$$

for some $\delta<\frac{1}{2}$. Then an easy covering argument (using (VD)) gives $\operatorname{CS}(\beta)$ with $\delta=\frac{1}{2}$.

2. Let $\lambda>1$. Suppose that $\operatorname{CS}(\beta)$ holds, except that instead of (3.1) we have

$$
\int_{B(x, s)} f^{2}|\nabla \varphi|^{2} d \mu \leq c_{2}(s / R)^{2 \theta}\left(\int_{B(x, \lambda s)}|\nabla f|^{2} d \mu+\psi(s)^{-1} \int_{B(x, \lambda s)} f^{2} d \mu\right) .
$$

Then once again it is easy to obtain $\operatorname{CS}(\beta)$ with $\lambda=2$ by a covering argument.

3. Any operation on the cut-off function $\varphi$ which reduces $|\nabla \varphi|$ while keeping properties (a), (b) and (c) of Definition 3.1 will generate a new cut-off function which still satisfies (3.1). We can therefore assume that any cut-off function $\varphi$ satisfies the following:

(a) $0 \leq \varphi \leq 1$.

(b) $\varphi$ is monotone on each cable.

(c) For each $t \in(0,1)$ the set $\{x: \varphi(x)>t\}$ is connected and contains $B\left(x_{0}, R / 2\right)$.

(d) Each connected component $A$ of $\{x: \varphi(x)<t\}$ intersects $B\left(x_{0}, R\right)^{c}$.

4. We call (3.1) a weighted Sobolev inequality. Note also that in order to prove (3.1) it is enough to consider nonnegative $f$.

Proposition 3.3. Suppose $C S(\beta)$ holds for $G$. Then it holds for $G_{C}$.

Proof. It is enough to prove this when $R$ and $s$ are both large. Let $x_{0} \in G_{C}$, and choose $x_{1} \in G$ with $d\left(x_{1}, x_{0}\right) \leq 1 / 2$. Let $\bar{\varphi}: G \rightarrow[0,1]$ be the cut-off function for $B\left(x_{1}, R\right)$. Define $\varphi: G_{C} \rightarrow[0,1]$ to be equal to $\bar{\varphi}$ on $G$ and by linear interpolation of $\bar{\varphi}$ on each cable.

Now let $I=B\left(x_{0}, s\right) \subset G_{C}$, with $s \geq 1$. We may assume $x_{0} \in G$ : if not, we prove the weighted Sobolev inequality for each of the balls $B\left(z_{i}, s\right)$, where $z_{i}$ are the endpoints of the cable containing $x_{0}$. We may also assume, again by using the remarks above, that $s=n+1 / 2$, where $n \in \mathbb{Z}$. Let $f: I^{*} \rightarrow \mathbb{R}_{+}$, where $I^{*}=B\left(x_{0}, 2 s\right)$.

For $x \in I^{*} \cap G$ define

$$
g(x)=V(x, 1 / 2)^{-1} \int_{B(x, 1 / 2)} f d \mu .
$$

Note that $V(x, 1 / 2)=\mu_{0}(x) / 2$ and that using the $\left(p_{0}\right)$ condition, if $x \sim y$, then $a_{x y} \leq \mu_{0}(x) \leq p_{0}^{-1} a_{x y}$. We have

$$
\int_{B(x, 1 / 2)} f^{2} d \mu=\int_{B(x, 1 / 2)}(f(y)-g(x))^{2} d \mu+\frac{1}{2} g(x)^{2} \mu_{0}(x) .
$$

It is elementary to prove the Poincaré inequality for $B(x, 1 / 2)$, and so

$$
\int_{B(x, 1 / 2)} f^{2} d \mu \leq c_{1} \int_{B(x, 1 / 2)}|\nabla f|^{2} d \mu+c_{1} g(x)^{2} \mu_{0}(x) .
$$


Since $\max _{y \sim x}|\bar{\varphi}(y)-\bar{\varphi}(x)|^{2} \leq \sum_{y \sim x}|\bar{\varphi}(y)-\bar{\varphi}(x)|^{2}$,

$$
\begin{aligned}
\int_{I} f^{2}|\nabla \varphi|^{2} d \mu & =\sum_{x \in I \cap G} \int_{B(x, 1 / 2)} f^{2}|\nabla \varphi|^{2} d \mu \\
& \leq c_{1} \sum_{x \in I \cap G}\left(\max _{y \sim x}|\bar{\varphi}(y)-\bar{\varphi}(x)|^{2}\right)\left(\int_{B(x, 1 / 2)}|\nabla f|^{2} d \mu+g(x)^{2} \mu_{0}(x)\right) \\
& \leq c_{2} R^{-2 \theta} \int_{I^{*}}|\nabla f|^{2} d \mu+c_{2} \sum_{x \in I \cap G} \sum_{y \sim x} a_{x y}|\bar{\varphi}(y)-\bar{\varphi}(x)|^{2} g(x)^{2} .
\end{aligned}
$$

We use the weighted Sobolev inequality for $g$ and $\bar{\varphi}$ to bound the second term in (3.3):

$$
\begin{aligned}
& c_{2} \sum_{x \in I \cap G} \sum_{y \sim x} a_{x y}|\bar{\varphi}(y)-\bar{\varphi}(x)|^{2} g(x)^{2} \\
& \quad \leq c_{3}(s / R)^{2 \theta}\left(\sum_{x \in I^{*}} \sum_{y \in I^{*}} a_{x y}|g(x)-g(y)|^{2}+s^{-\beta} \sum_{x \in I^{*}} g(x)^{2} \mu_{0}(x)\right) .
\end{aligned}
$$

We now bound the terms in (3.4) by the corresponding expressions involving $f$. By Jensen's inequality $g(x)^{2} \leq V(x, 1 / 2)^{-1} \int_{B(x, 1 / 2)} f^{2} d \mu$, and so

$$
\sum_{x \in I^{*}} g(x)^{2} \mu_{0}(x) \leq \int_{I^{*}} f^{2} d \mu .
$$

If $x, y \in I^{*}$ with $x \sim y$, then there exist $x^{\prime} \in B(x, 1 / 2), y^{\prime} \in B(y, 1 / 2)$ with $|g(x)-g(y)| \leq\left|f\left(x^{\prime}\right)-f\left(y^{\prime}\right)\right|$. So, writing $d t$ for the measure on $G_{C}$ which equals Lebesgue measure on each cable,

$$
|g(x)-g(y)| \leq \int_{B(x, 1 / 2) \cup B(y, 1 / 2)}|\nabla f(t)| d t \leq c_{4}\left(\int_{B(x, 1 / 2) \cup B(y, 1 / 2)}|\nabla f(t)|^{2} d t\right)^{1 / 2} .
$$

Writing $A(x)=\int_{B(x, 1 / 2)}|\nabla f|^{2} d t$, we have $A(x) \mu_{0}(x) \leq c_{5} \int_{B(x, 1 / 2)}|\nabla f|^{2} d \mu$. So,

$$
\begin{aligned}
\sum_{x \in I^{*} \cap G} \sum_{y \in I^{*} \cap G} a_{x y}|g(x)-g(y)|^{2} & \leq c_{6} \sum_{x \in I^{*} \cap G} \sum_{y \in I^{*} \cap G} a_{x y}(A(x)+A(y)) \\
& =2 c_{6} \sum_{x \in I^{*} \cap G} A(x) \mu_{0}(x) \\
& \leq c_{7} \sum_{x \in I^{*} \cap G} \int_{B(x, 1 / 2)}|\nabla f|^{2} d \mu \\
& \leq c_{8} \int_{I^{*}}|\nabla f|^{2} d \mu .
\end{aligned}
$$

Combining (3.3)-(3.6) we deduce (3.1) for $f$.

Proposition 3.4. Suppose $C S(\beta)$ holds for $G_{C}$. Then it holds for $G$.

Proof. Let $x_{0} \in G$, and $R \geq 1$. Let $\varphi: G_{C} \rightarrow[0,1]$ be a cut-off function for $B\left(x_{0}, R\right)$. Now let $x_{1} \in G, 1 \leq s \leq R$ and $I=B\left(x_{1}, s\right)$. Let $g: I^{*} \cap G \rightarrow \mathbb{R}_{+}$. Extend $g$ to a function $f: I^{*} \rightarrow \mathbb{R}_{+}$by linear interpolation on cables. We take $f$ to be constant on any cable with only one end in $I^{*}$. 
If $e$ is the edge $\{x, y\}$ with $x, y \in I$, write $C_{e}$ for the cable associated with $e, a_{e}=$ $a_{x y}, g(e)^{2}=\left(g(x)^{2}+g(y)^{2}\right) / 2,|\nabla \varphi(e)|=|\varphi(x)-\varphi(y)|$, and $|\nabla g(e)|=|g(x)-g(y)|$. Let $E_{0}=\{e=\{x, y\}: x, y \in I\}$,

$$
E_{1}=\left\{e=\{x, y\} \in E_{0}: \frac{1}{2} g(x) \leq g(y) \leq 2 g(x)\right\},
$$

and $E_{2}=E_{0}-E_{1}$. If $e \in E_{2}$, then an elementary computation shows $g(e)^{2} \leq$ $3|\nabla g(e)|^{2}$. So by Definition 3.1(c),

$$
\sum_{e \in E_{2}} a_{e} g(e)^{2}|\nabla \varphi(e)|^{2} \leq c_{1} R^{-2 \theta} \sum_{e \in E_{2}} a_{e}|\nabla g(e)|^{2} .
$$

If $e \in E_{1}$ and $g(x) \leq g(y)$, then we have $g(e)^{2} \leq 5 g(x)^{2}$, and so

$$
a_{e} g(e)^{2}|\nabla \varphi(e)|^{2} \leq c_{2} g(x)^{2} \int_{C_{e}}|\nabla \varphi|^{2} d \mu \leq c_{2} \int_{C_{e}} f^{2}|\nabla \varphi|^{2} d \mu .
$$

Hence, using the weighted Sobolev inequality for $f$ and $\varphi$,

$$
\begin{aligned}
\sum_{e \in E_{1}} a_{e} g(e)^{2}|\nabla \varphi(e)|^{2} & \leq c_{3} \int_{I} f^{2}|\nabla \varphi|^{2} d \mu \\
& \leq c_{4}(s / R)^{2 \theta}\left(\int_{I^{*}}|\nabla f|^{2} d \mu+s^{-\beta} \int_{I^{*}} f^{2} d \mu\right) .
\end{aligned}
$$

Note that $|\nabla f|=|g(x)-g(y)|$ on $C_{e}$, and so

$$
\int_{I^{*}}|\nabla f|^{2} d \mu \leq \sum_{x \in I^{*}} \sum_{y \in I^{*}} a_{x y}|g(x)-g(y)|^{2} .
$$

We bound $\int_{I^{*}} f^{2} d \mu$ as in the previous result. Combining (3.7), (3.8) with these bounds we obtain the weighted Sobolev inequality for $g$.

We will also need the equivalence of the Poincaré inequality for $G$ and for $G_{C}$.

Proposition 3.5. (a) Suppose $(G, E, a)$ satisfies $P I(\beta)$. Suppose the gradient of $f$ is square integrable over $B(x, r)$ and $r>0$. Then, writing $f_{B}=\mu(B)^{-1} \int_{B} f d \mu$,

$$
\int_{B(x, r)}\left|f(z)-f_{B(x, r)}\right|^{2} \mu(d z) \leq c_{1} \psi(r) \int_{B(x, r)}|\nabla f(z)|^{2} \mu(d z) .
$$

(b) Conversely, if (3.9) holds for every $x$ and $r$ with $c_{1}$ independent of $x$ and $r$, then $(G, E, a)$ satisfies $P I(\beta)$.

The proof of Proposition 3.5 is very similar to the proofs of Propositions 3.3 and 3.4. Similarly we have

Proposition 3.6. $G$ satisfies $\left(R_{\beta}\right)$ if and only if $G_{C}$ satisfies $\left(R_{\beta}\right)$.

\section{Construction of Cut-off functions}

In this section we will assume that the conditions (a)-(d) of Theorem 1.2 hold and prove that both $P I(\beta)$ and $\operatorname{CS}(\beta)$ follow.

Lemma 4.1. Suppose $(G, E, a)$ satisfies $P H I(\beta)$. Then $(G, E, a)$ satisfies $P I(\beta)$.

Proof. Using the estimates (1.12) and (1.13), the Poincaré inequality for $G$ follows by the argument given in $[\underline{\mathrm{SC}}$. 
For the remainder of this section we work on the cable system $G_{C}$ associated with the graph $G$. If $D$ is a domain in $G_{C}$ (so $D \subset G_{C}, D$ is connected and relatively open in $\left.G_{C}\right)$, write $g_{D}(x, y)$ for the Green function of $Y$ on $D$. Then $g_{D}$ is symmetric and continuous, and we have for $f \in \mathcal{D}(\mathcal{L})$ with support contained in $D$

$$
\mathcal{E}\left(g_{D}(x, \cdot), f\right)=-\int_{D} g_{D}(x, y) \mathcal{L} f(y) \mu(d y)=f(x) ;
$$

see [FOT], Corollary 1.3.1. We have $g_{D}(x, y)=0$ if $y \in \partial D$, so we can extend $g_{D}$ to $G_{C} \times G_{C}$ by taking it to be zero off $D \times D$. We write $g=g_{G_{C}}$ when this exists. Note that if $x \in G, B(x, 1) \subset D$, then applying (4.1) to a function $f$ with $f=1$ on $B(x, 1 / 2)$ and $f=0$ off $B(x, 1)$, then

$$
\frac{1}{2} \sum_{y \in G} a_{x y}\left(g_{D}(x, x)-g_{D}(x, y)\right)=1 .
$$

The following lemma is a slight generalization of Proposition 4.1 of GT2 and is proved similarly; note that no geometric assumptions on $(G, E)$ are needed.

Lemma 4.2. Suppose $(G, E, a)$ satisfies (EHI). Then there exists a constant $c_{1}$ such that if $x_{0} \in G_{C}, r>0, d\left(x_{0}, x\right)=d\left(x_{0}, y\right)=r$, and $B\left(x_{0}, 2 r\right) \subset D$, then

$$
c_{1}^{-1} g_{D}\left(x_{0}, y\right) \leq g_{D}\left(x_{0}, x\right) \leq c_{1} g_{D}\left(x_{0}, y\right) .
$$

The following result is proved in [GT2] and [T1], but as it has a simple direct proof which does not use (EHI), we present it here.

Lemma 4.3. Suppose $(G, E)$ satisfies $(V D)$ and $\left(E_{\beta}\right)$. Then $G$ satisfies $\left(R_{\beta}\right)$.

Proof. Write $I$ for $B\left(x_{0}, r\right)$ and $I^{*}$ for $B\left(x_{0}, 2 r\right)$. Let $f$ be the function which is 1 on $I$ and zero on $\left(I^{*}\right)^{c}$ and for which the infimum in (1.10) is attained. $f$ will be bounded between 0 and 1 . Then it is well known that

$$
f(x)=\int_{I} g_{I^{*}}(z, x) e_{I}(d z),
$$

where $e_{I}$ is the capacitary measure for $I$ (relative to $g_{I^{*}}$ ), and also that the effective resistance between $\partial I$ and $\partial I^{*}$ is exactly $e_{I}(I)^{-1}$. For $z \in I$ we have,

$$
r^{\beta} \approx \mathbb{E}^{z} \tau_{I^{*}}=\int_{I^{*}} g_{I^{*}}(z, x) \mu(d x) .
$$

Using (VD) we have

$$
V\left(x_{0}, r\right)=\int_{I} f(x) \mu(d x) \leq \int_{I^{*}} f(x) \mu(d x) \leq V\left(x_{0}, 2 r\right) \leq c_{1} V\left(x_{0}, r\right),
$$

and also

$$
\begin{aligned}
\int_{I^{*}} f(x) \mu(d x) & =\int_{I^{*}}\left(\int_{I} g_{I^{*}}(z, x) e_{I}(d z)\right) \mu(d x) \\
& =\int_{I} \mathbb{E}^{z}\left(\tau_{I^{*}}\right) e_{I}(d z) \approx e_{I}(I) r^{\beta} .
\end{aligned}
$$

Rearranging the final formula gives the result.

From now on in this section we assume that $G_{C}$ satisfies (VD), (EHI) and $\left(E_{\beta}\right)$ and (FVG) for some $\alpha_{1}>\beta$. 
Proposition 4.4. Suppose $G_{C}$ satisfies (VD), (EHI), $\left(E_{\beta}\right)$, and also (FVG) for some $\alpha_{1}>\beta$.

(a) If $x, y \in G_{C}$ with $d(x, y)=r \geq 1$, and $D \subset G_{C}$ with $B(x, 2 r) \subset D$, then

$$
g_{D}(x, y) \approx \frac{r^{\beta}}{V(x, r)} .
$$

In particular, $(G, E, a)$ is transient.

(b) If $x, y \in G_{C}$ with $d(x, y)=r \leq 1$, and $D \subset G_{C}$ with $B(x, 2) \subset D$, then $g_{D}(x, y) \approx V(x, 1)^{-1}$.

Proof. (a) Write $B_{k}=B\left(x, 2^{k} r\right)$ for $k \geq 0$. If $B_{n} \subset D$, then by GT2, Proposition 4.4,

$$
g_{D}(x, y) \approx \sum_{k=0}^{n-1} R_{\mathrm{eff}}\left(B_{k}, B_{k+1}^{c}\right) .
$$

(We can use Lemmas 4.2 and 4.3 to replace the constant $M$ in [GT2] by 2.) So by Lemma 4.3,

$$
g_{D}(x, y) \approx \sum_{k=0}^{n-1} \frac{\left(2^{k} r\right)^{\beta}}{V\left(x, 2^{k} r\right)}=\frac{r^{\beta}}{V(x, r)} \sum_{k=0}^{n-1} 2^{k \beta} \frac{V(x, r)}{V\left(x, 2^{k} r\right)} .
$$

This immediately implies that if $B_{2} \subset D$, then $g_{D}(x, y) \geq 2^{\beta} r^{\beta} / V(x, r)$. Since $\alpha_{1}>\beta$ the condition (FVG) implies

$$
\sum_{k=0}^{n-1} 2^{k \beta} \frac{V(x, r)}{V\left(x, 2^{k} r\right)} \leq c_{1} \sum_{k=0}^{\infty} 2^{\left(\beta-\alpha_{1}\right) k} \leq c_{2},
$$

proving the upper bound.

Taking $D=G_{C}$ we see that $g(x, y)<\infty$, so that $G$ is transient.

(b) Choose $z$ with $d(x, z)=1$; by (a) $g_{D}(x, z) \approx V(x, 1)^{-1}$. Using the local Harnack inequality and (4.2) completes the proof.

Let $x_{0} \in G_{C}$. Define

$$
Q(h)=Q\left(x_{0}, h\right)=\left\{y: g\left(x_{0}, y\right)>h\right\} .
$$

Clearly, if $h_{1}<h_{2}$, then $Q\left(h_{2}\right) \subset Q\left(h_{1}\right)$. Since $g\left(x_{0}, \cdot\right)$ is harmonic except at $x_{0}$, it is linear on each cable not containing $x_{0}$. On the cable containing $x_{0}$, it has its maximum at $x_{0}$ and is monotonically decreasing as a function of the distance to $x_{0}$ on each side of $x_{0}$. Therefore the intersection of $Q(h)$ with any cable will consist of at most one interval. Also, by the maximum principle, we have that $Q(h)$ is connected. The next lemma proves that the sets $Q(h)$ can be approximated from within and without by balls.

Given $x_{0} \in G_{C}, R>0$ define

$$
h=h\left(x_{0}, R\right)=\sup _{B\left(x_{0}, R\right)^{c}} g\left(x_{0}, \cdot\right) .
$$

Lemma 4.5. Suppose $G_{C}$ satisfies (VD), $\left(E_{\beta}\right),(E H I)$, and also ( $\left.F V G\right)$ for some $\alpha_{1}>\beta$.

(a) There exists $\sigma \in(2, \infty)$, independent of $x_{0}$ and $R$, such that if $R \geq \sigma$, then

$$
B\left(x_{0}, \sigma^{-1} R\right) \subset Q(2 h) \subset Q(h) \subset B\left(x_{0}, \sigma R\right) .
$$


(b) Provided that $t R \geq 1$,

$$
h\left(x_{0}, t R\right) \leq c_{1} t^{\beta-\alpha_{1}} h\left(x_{0}, R\right) .
$$

Proof. (a) Note that $Q(h) \subset B\left(x_{0}, R\right)$ from (4.6). Let $t \in(0,1)$ and $y \in B\left(x_{0}, t R\right)$. Then provided $t R \geq 1$, using (4.5) and (FVG)

$$
g\left(x_{0}, y\right) \geq c_{2} \frac{t^{\beta} R^{\beta}}{V\left(x_{0}, t R\right)} \geq c_{3} \frac{t^{\beta-\alpha_{1}} R^{\beta}}{V\left(x_{0}, R\right)} \geq c_{4} t^{\beta-\alpha_{1}} h .
$$

Define $\sigma$ by $c_{4} \sigma^{\alpha_{1}-\beta}=2$. Then we deduce that $B\left(x_{0}, \sigma^{-1} R\right) \subset Q(2 h)$ provided $R \geq \sigma$.

(b) follows in a similar fashion from (4.5) and (VD).

Note that if $u$ is harmonic in a domain $D$ and the elliptic Harnack inequality holds, then there exists $\rho<1$ independent of $x_{0}$ and $r$ such that

$$
\operatorname{Osc}_{B\left(x_{0}, r\right)} u \leq \rho \operatorname{Osc}_{B\left(x_{0}, 2 r\right)} u \text {. }
$$

Here $\operatorname{Osc}_{A} u=\sup _{A} u-\inf _{A} u$. This is standard - see [M1], Section 5, for example.

Lemma 4.6. Let $x_{0}, \rho$, and $R$ be as above, let $\theta=-\log \rho / \log 2$, and let $h$ be defined by (4.6). Let $x, y \in B\left(x_{0}, R / 2 \sigma\right)^{c}$ with $d(x, y)=s$. Then

$$
\left|g\left(x_{0}, x\right)-g\left(x_{0}, y\right)\right| \leq c_{1}(s / R)^{2 \theta} h\left(x_{0}, R\right) .
$$

Proof. First note that $g\left(x_{0}, x\right)$ and $g\left(x_{0}, y\right)$ are both bounded by $c_{2} h$, so the result is clear if $s \geq R / 4 \sigma$. So suppose $s<R / 4 \sigma$, let $n$ (with $n \geq 0$ ) be the largest integer so that $B\left(x_{0}, R / 4 \sigma\right) \cap B\left(x, 2^{n} s\right)=\emptyset$, and write $a_{k}=\operatorname{Osc}_{B\left(x, 2^{k} s\right)} g\left(x_{0}, \cdot\right)$. Then $a_{n} \leq c_{2} h$, and using (4.8) we have $a_{k} \leq \rho a_{k+1}$, where $\rho<1$ is a constant independent of $k$ and $x_{0}$. Since $R / 4 \sigma<2^{n+1} s$, then

$$
\left|g\left(x_{0}, x\right)-g\left(x_{0}, y\right)\right| \leq a_{0} \leq c_{3} h \rho^{n}=c_{4} h\left(2^{n}\right)^{-\theta} \leq c_{5} h(s / R)^{\theta} .
$$

Let $D$ be a domain in $G_{C}$, and $A \subset D$. Define

$$
U(x, A, D)=\int_{A} g_{D}(x, y) \mu(d y)=\mathbb{E}^{x} \int_{0}^{\tau_{D}} 1_{A}\left(Y_{s}\right) d s .
$$

Note that $U$ is monotone in $A$ and $D$ : if $A \subset A^{\prime} \subset D \subset D^{\prime}$, then

$$
U(x, A, D) \leq U\left(x, A^{\prime}, D\right) \leq U\left(x, A^{\prime}, D^{\prime}\right) .
$$

Lemma 4.7. Suppose (VD), $\left(E_{\beta}\right),(E H I)$, and (FVG) hold. We have

$$
\begin{aligned}
U\left(x, B\left(x_{0}, r\right), B\left(x_{0}, r\right)\right) & \leq c_{1} \psi(r) \text { for } x \in G, \\
U\left(x, B\left(x_{0}, r\right), B\left(x_{0}, 2 r\right)\right) & \geq c_{2} \psi(r) \text { for } x \in B\left(x_{0}, r\right) .
\end{aligned}
$$

Proof. The result is easy if $r<1$, so we take $r \geq 1$. If $x \notin B\left(x_{0}, r\right)$, the left hand side of (a) is 0 . If $x \in B\left(x_{0}, r\right)$ we have $\tau_{B\left(x_{0}, r\right)} \leq \tau_{B(x, 2 r)}$, so

$$
U\left(x, B\left(x_{0}, r\right), B\left(x_{0}, r\right)\right)=\mathbb{E}^{x} \tau_{B\left(x_{0}, r\right)} \leq \mathbb{E}^{x} \tau_{B(x, 2 r)} \leq c_{3} r^{\beta},
$$

proving (a).

(b) Suppose $x \in B\left(x_{0}, r\right)$. Using Proposition 4.4 we deduce that if $y \in B\left(x_{0}, r\right)$ with $r \geq d(x, y)>r / 6$, then $g_{B\left(x_{0}, 2 r\right)}(x, y) \geq c_{4} r^{\beta} / V(x, r)$. Since for any $x \in$ 
$B\left(x_{0}, r\right)$ there exists a ball $B\left(x^{\prime}, r / 3\right) \subset B\left(x_{0}, r\right)$ with $x \notin B\left(x^{\prime}, r / 3\right)$, it follows that

$$
\begin{aligned}
U\left(x, B\left(x_{0}, r\right), B\left(x_{0}, 2 r\right)\right) & =\int_{B\left(x_{0}, r\right)} g_{B\left(x_{0}, 2 r\right)}(x, y) \mu(d y) \\
& \geq \int_{B\left(x^{\prime}, r / 6\right)} g_{B\left(x_{0}, 2 r\right)}(x, y) \mu(d y) \geq r^{\beta} \frac{V\left(x^{\prime}, r / 6\right)}{V(x, r)} \geq c_{5} r^{\beta},
\end{aligned}
$$

where we used (VD) in the final line.

Lemma 4.8. Let $D$ be a domain in $G_{C}$, and $A \subset D$, and $v=U(\cdot, A, D)$. Suppose that $\partial A \cap G=\emptyset$, and the intersection of $\partial A$ with each cable is a finite set. Then if $f \in \mathcal{D}\left(\mathcal{E}_{Y}\right)$,

$$
\int_{D} \nabla\left(f^{2} v\right) \cdot \nabla v d \mu=\int_{A} f^{2} v d \mu .
$$

Proof. Note that $v \in \mathcal{K}(D)$ and $\mathcal{L} v=-1_{A}$. Suppose first $\partial D \cap G=\emptyset$. Then, by Lemma 2.3,

$$
\int_{D} \nabla\left(f^{2} v\right) \cdot \nabla v d \mu=\int_{A} f^{2} v d \mu-\sum_{z \in \partial D} a_{e(z)} f^{2}(z) v(z) \frac{\partial v}{\partial n}(z) .
$$

As $v(z)=0$ on $\partial D$ we obtain (4.9). If $\partial D \cap G$ is non-empty, we can now obtain (4.9) by an approximation argument.

Now let $x_{0} \in G_{C}$ and $R>0$. We will construct a cut-off function $\varphi$ for $B\left(x_{0}, R\right)$. If $R \leq c_{1}$ this is easy, so we assume $R>c_{1}$. Define $h$ by (4.6), so that, by Proposition 4.4 and Lemma $4.5, h \approx R^{\beta} / V\left(x_{0}, R\right)$ and (4.7) holds. Let

$$
\begin{aligned}
\omega_{0}(x) & =g\left(x_{0}, x\right), \quad x \in G_{C}, \\
\omega(x) & =\left(2 h \wedge g\left(x_{0}, x\right)-h\right)^{+}, \quad x \in G_{C} .
\end{aligned}
$$

We use the following elementary result.

Lemma 4.9. Let $x, y, z \geq 0$. If $x \leq c_{1}\left(x^{1 / 2} z^{1 / 2}+y\right)$, then $x \leq 2 c_{1} y+4 c_{1}^{2} z$.

Proposition 4.10. Let $x_{0}, R, \omega, \omega_{0}$ be as above. Let $I=B(x, s)$, with $s \leq R$. Suppose that either

$$
I^{*} \subset Q(2 h)
$$

or

$$
I^{*} \cap B\left(x_{0}, R / 2 \sigma\right)=\emptyset .
$$

Suppose $f$ and its gradient are square integrable over $I^{*}$. There exists $c_{1}<\infty$ such that

$$
\int_{I} f^{2}|\nabla \omega|^{2} \leq c_{1}(s / R)^{2 \theta} h^{2}\left(\int_{I^{*}}|\nabla f|^{2}+\psi(s)^{-1} \int_{I} f^{2}\right) .
$$

Proof. If (4.11) holds, then $\nabla \omega=0$ on $I^{*}$, and the left hand side of (4.13) is 0 . So we suppose (4.12) holds. Let $h_{1}=h\left(x_{0}, R / 2 \sigma\right)$; then $h_{1} \leq c_{2} h$ and $I^{*} \subset Q\left(h_{1}\right)^{c}$. Let $v=U\left(\cdot, I, I^{*}\right)$, and write $V_{0}=\inf _{I} v, V_{1}=\sup _{I^{*}} v$. By Lemma 4.7 we have

$$
c_{3} \psi(s) \leq V_{0} \leq V_{1} \leq c_{4} \psi(s) .
$$

Fix $x_{1} \in I$ and set $\omega_{1}=\omega_{0}-\omega_{0}\left(x_{1}\right)$. Let $H$ be a domain such that $\overline{I^{*}} \subset H \subset \bar{H} \subset$ $Q\left(2 h_{1}\right)^{c}$ and $(\partial H) \cap G=\emptyset$. Since $H \subset Q\left(2 h_{1}\right)^{c}$, then $\omega_{0} \leq 2 h_{1}$ on $H$, and hence 
$\left|\omega_{1}\right| \leq 2 c_{2} h$ on $H$. On the other hand, $\left|\omega_{1}\right|$ is bounded on $I^{*}$ by $\operatorname{Osc}_{I^{*}} \omega_{0}$. So by Lemma 4.6,

$$
L=\sup _{I^{*}}\left|\omega_{1}\right| \leq \operatorname{Osc}_{B(x, 2 s)} \omega_{0} \leq c_{5} h(s / R)^{\theta} .
$$

Write

$$
\begin{aligned}
& A=\int_{I^{*}} f^{2} v^{2}|\nabla \omega|^{2}, \\
& B=\int_{I^{*}} v^{2}|\nabla f|^{2}, \\
& C=\int_{I} f^{2}, \\
& D=\int_{I^{*}} f^{2}|\nabla v|^{2}, \\
& E=\int_{I^{*}}|\nabla f|^{2}, \\
& F=\int_{H} f^{2} v^{2}\left|\nabla \omega_{1}\right|^{2}=\int_{I^{*}} f^{2} v^{2}\left|\nabla \omega_{1}\right|^{2} .
\end{aligned}
$$

Observe that

$$
\int_{I} f^{2}|\nabla \omega|^{2} \leq\left(\inf _{I} v\right)^{-2} \int_{I} f^{2}|\nabla \omega|^{2} v^{2} \leq V_{0}^{-2} A .
$$

Also, since $v$ is 0 on $\left(I^{*}\right)^{c}$,

$$
\begin{aligned}
A & =\int_{I^{*}} f^{2} v^{2}|\nabla \omega|^{2}=\int_{H} f^{2} v^{2}|\nabla \omega|^{2} \\
& \leq \int_{H} f^{2} v^{2}\left|\nabla \omega_{0}\right|^{2}=\int_{H} f^{2} v^{2}\left|\nabla \omega_{1}\right|^{2}=F .
\end{aligned}
$$

We work first on bounding $F$. By Lemma 2.3,

$$
\begin{aligned}
F & =\int_{H} f^{2} v^{2} \nabla \omega_{1} \cdot \nabla \omega_{1}=\int_{H} \nabla\left(\omega_{1} f^{2} v^{2}\right) \cdot \nabla \omega_{1}-\int_{H} \omega_{1} \nabla\left(f^{2} v^{2}\right) \cdot \nabla \omega_{1} \\
& =-\int_{H} \omega_{1} f^{2} v^{2} \mathcal{L} \omega_{1}-\sum_{\partial H} a_{e(\cdot)} \omega_{1} f^{2} v^{2} \frac{\partial \omega_{1}}{\partial n}-\int_{H} \omega_{1} \nabla\left(f^{2} v^{2}\right) \cdot \nabla \omega_{1} .
\end{aligned}
$$

As $H \cap Q\left(2 h_{1}\right)=\emptyset$, then $\mathcal{L} \omega_{1}=0$ in $H$, while $v=0$ on $\partial H$. Hence

$$
F=-\int \omega_{1} \nabla\left(f^{2} v^{2}\right) \cdot \nabla \omega_{1} .
$$

We then proceed to bound $F$ by writing

$$
\begin{aligned}
F & \leq\left|\int_{H} \omega_{1} \nabla \omega_{1} \cdot\left(2 f^{2} v \nabla v+2 f v^{2} \nabla f\right)\right| \\
& \leq 2\left|\int_{H} f v \omega_{1} \nabla \omega_{1} \cdot(f \nabla v)\right|+2\left|\int_{H} f v \omega_{1} \nabla \omega_{1} \cdot(v \nabla f)\right| \\
& \leq 2\left(\int_{H} f^{2} v^{2} \omega_{1}^{2}\left|\nabla \omega_{1}\right|^{2}\right)^{1 / 2}\left[\left(\int_{H} f^{2}|\nabla v|^{2}\right)^{1 / 2}+\left(\int_{H} v^{2}|\nabla f|^{2}\right)^{1 / 2}\right] \\
& =2\left(B^{1 / 2}+D^{1 / 2}\right)\left(\int_{H} \omega_{1}^{2} f^{2} v^{2}\left|\nabla \omega_{1}\right|^{2}\right)^{1 / 2} .
\end{aligned}
$$


Then as $v=0$ on $\left(I^{*}\right)^{c}$ and $\left|\omega_{1}\right| \leq L$ on $I^{*}$,

$$
F \leq 2\left(B^{1 / 2}+D^{1 / 2}\right) L F^{1 / 2}
$$

and dividing both sides by $F^{1 / 2}$,

$$
F \leq c_{6} L^{2}\left(B^{1 / 2}+D^{1 / 2}\right)^{2} \leq c_{7} L^{2}(B+D) .
$$

We now bound $D$. We have, using Lemma 4.8

$$
\begin{aligned}
D & =\int_{H} f^{2} \nabla v \cdot \nabla v=\int_{H} \nabla\left(f^{2} v\right) \cdot \nabla v-\int_{H} 2 f v \nabla f \cdot \nabla v \\
& =\int_{I} f^{2} v-\int_{H} 2 f v \nabla f \cdot \nabla v \\
& \leq \int_{I} f^{2} v+c_{8}\left(\int_{I^{*}} f^{2}|\nabla v|^{2}\right)^{1 / 2}\left(\int_{I^{*}} v^{2}|\nabla f|^{2}\right)^{1 / 2} \\
& \leq c_{9} V_{1} C+c_{9} B^{1 / 2} D^{1 / 2} .
\end{aligned}
$$

Therefore by Lemma 4.9

$$
D \leq c_{10}\left(B+c_{11} V_{1} C\right)
$$

Since

we obtain

$$
B \leq V_{1}^{2} \int_{I^{*}}|\nabla f|^{2}=V_{1}^{2} E,
$$

$$
A \leq F \leq c_{12} L^{2}(B+D) \leq c_{13} L^{2}\left(V_{1} C+V_{1}^{2} E\right) .
$$

We have $V_{1} / V_{0}$ bounded independently of $s$. Since $L \leq c_{14} h(s / R)^{\theta}$, our result follows using (4.14).

Corollary 4.11. Let $(G, E, a)$ satisfy $P H I(\beta)$ and $F V G$ for some $\alpha_{1}>\beta$. Then $G$ and $G_{C}$ satisfy $C S(\beta)$ for some $\theta>0$.

Proof. Let $x_{0} \in G_{C}$ and $R>0$, let $\omega$ be given by (4.10), and let $\varphi=1 \wedge$ $\left(h\left(x_{0}, R\right)^{-1} \omega\right)$. It is clear that $\varphi=1$ on $B\left(x_{0}, R / \sigma\right)$ and that $\varphi=0$ outside $B\left(x_{0}, R\right)$. By Lemma $4.6 \varphi$ satisfies Definition 3.1(c).

It remains to prove that $\varphi$ satisfies the weighted Sobolev inequality (3.1). Let $I=B(x, s)$ with $s \leq R$. If $I^{*}$ satisfies either (4.11) or (4.12), then, using Proposition 4.10 , we are done. If $I^{*}$ fails to satisfy both, then $I^{*}$ must intersect both $B\left(x_{0}, R / 2 \sigma\right)$ and $B\left(x_{0}, R / \sigma\right)^{c}$, and so $s \geq R / 8 \sigma$.

We use Lemma 2.7 to cover $I$ with balls $B_{i}=B\left(x_{i}, c_{1} R\right)$, where $c_{1} \in(0,1 / 4 \sigma)$ has been chosen small enough so that each $B_{i}^{*}$ satisfies at least one of (4.11) or (4.12). We can then apply (4.13) with $I$ replaced by each ball $B_{i}$ : writing $s^{\prime}=c_{1} R$ we have

$$
\int_{B_{i}} f^{2}|\nabla \varphi|^{2} \leq c_{2}\left(s^{\prime} / R\right)^{2 \theta}\left(\int_{B_{i}^{*}}|\nabla f|^{2}+\psi\left(s^{\prime}\right)^{-1} \int_{B_{i}^{*}} f^{2}\right) .
$$

We then sum over $i$. Since no point of $I^{*}$ is in more than $M$ (not depending on $x_{0}$, $R$ or $h$ ) of the $B_{i}^{*}$, and $s / c_{1} \leq s^{\prime} \leq s$, we obtain (3.1) for $I$.

It remains to remove the hypothesis (FVG), which we do by a trick involving products.

Theorem 4.12. Suppose $(G, E, a)$ satisfies $P H I(\beta)$. Then there exists $\theta>0$ such that $G$ satisfies $C S(\beta)$. 
Proof. Choose $\alpha_{1}>\beta$. By Theorem 2 of B1] there exists a graph $\left(G^{\prime}, E^{\prime}, a^{\prime}\right)$ satisfying $\left(p_{0}\right),(\mathrm{EHI})$ and $\left(E_{\beta}\right)$ and with $V^{\prime}(x, r) \approx r^{\alpha_{1}}$, and $a_{x^{\prime} y^{\prime}}^{\prime}$ equal to 0 or 1. We may also assume that $\left\{x^{\prime}, x^{\prime}\right\} \in E^{\prime}$ for all $x^{\prime} \in G^{\prime}$. Write $p_{n}^{\prime}\left(x^{\prime}, y^{\prime}\right)$ for the transition density of the random walk $X^{\prime}$ on $\left(G^{\prime}, E^{\prime}\right)$. Since $\left\{y^{\prime}, y^{\prime}\right\} \in E^{\prime}$, we have $p_{n+1}^{\prime}\left(x^{\prime}, y^{\prime}\right) \geq c_{1} p_{n}^{\prime}\left(x^{\prime}, y^{\prime}\right)$ for some $c_{1}>0$. By Theorem $1.2\left(G^{\prime}, E^{\prime}, a^{\prime}\right)$ satisfies $\operatorname{PHI}(\beta)$, and also $p_{n}^{\prime}\left(x^{\prime}, y^{\prime}\right)$ satisfies the estimates (1.12) and (1.13). Further, the lower bound (1.13) holds for $p_{n}^{\prime}$, and not just $p_{n}^{\prime}+p_{n+1}^{\prime}$.

We now define the product graph $\widetilde{G}=G \times G^{\prime}$, and say that $\left\{(x, y),\left(x^{\prime}, y^{\prime}\right)\right\} \in \widetilde{E}$ if and only if $\left\{x, x^{\prime}\right\} \in E$ and $\left\{y, y^{\prime}\right\} \in E^{\prime}$. Define weights on $(\widetilde{G}, \widetilde{E})$ by

$$
\widetilde{a}_{\left(x, x^{\prime}\right),\left(y, y^{\prime}\right)}= \begin{cases}a_{x y} a_{x^{\prime} y^{\prime}}^{\prime} & \text { if }\left\{x, x^{\prime}\right\},\left\{y, y^{\prime}\right\} \in E^{\prime}, \\ 0 & \text { otherwise. }\end{cases}
$$

The graph $\widetilde{G}$ is connected, since $G^{\prime}$ is not bipartite.

Write $\widetilde{V}$ and $\widetilde{d}$ for the volume and distance, respectively, on $\widetilde{G}$. It is easy to check that

$$
\widetilde{V}\left(\left(x, x^{\prime}\right), R\right) \approx V(x, R) V^{\prime}\left(x^{\prime}, R\right) \approx R^{\alpha_{1}} V(x, R),
$$

so that $(\widetilde{G}, \widetilde{E})$ satisfies (VD) and (FVG) with the exponent $\alpha_{1}>\beta$. Because the weights on $(\widetilde{G}, \widetilde{E})$ are given as products, the Markov chain on $\widetilde{G}=G \times G^{\prime}$ is given by $\widetilde{X}=\left(X, X^{\prime}\right)$, where $X$ is the Markov chain on $G$ with weights $a$ and $X^{\prime}$ is the Markov chain on $G^{\prime}$; moreover $X$ and $X^{\prime}$ are independent. It follows that the transition densities for $\widetilde{X}$ are given as a product: we have $\widetilde{p}_{n}\left(\left(x, x^{\prime}\right),\left(y, y^{\prime}\right)\right)=$ $p_{n}(x, y) p_{n}^{\prime}\left(x^{\prime}, y^{\prime}\right)$. From Theorem 1.2 we deduce that

$$
\begin{aligned}
\widetilde{p}_{n}\left(\left(x, x^{\prime}\right),\left(y, y^{\prime}\right)\right) \leq & c_{2} V\left(x, n^{1 / \beta}\right)^{-1} V^{\prime}\left(x^{\prime}, n^{1 / \beta}\right)^{-1} \\
& \times \exp \left(-c_{3}\left(\frac{d(x, y)^{\beta}}{n}\right)^{1 /(\beta-1)}-c_{3}\left(\frac{d^{\prime}\left(x^{\prime}, y^{\prime}\right)^{\beta}}{n}\right)^{1 /(\beta-1)}\right) \\
\leq & c_{4} \widetilde{V}\left(\left(x, x^{\prime}\right), n^{1 / \beta}\right)^{-1} \exp \left(-c_{5}\left(\frac{\widetilde{d}\left(\left(x, x^{\prime}\right),\left(y, y^{\prime}\right)\right)^{\beta}}{n}\right)^{1 /(\beta-1)}\right) .
\end{aligned}
$$

Similarly we have if $n \geq \widetilde{d}\left(\left(x, x^{\prime}\right),\left(y, y^{\prime}\right)\right) \vee 1$,

$$
\begin{aligned}
\widetilde{p}_{n}\left(\left(x, x^{\prime}\right),\right. & \left.\left(y, y^{\prime}\right)\right)+\widetilde{p}_{n+1}\left(\left(x, x^{\prime}\right),\left(y, y^{\prime}\right)\right) \\
& =p_{n}(x, y) p_{n}^{\prime}\left(x^{\prime}, y^{\prime}\right)+p_{n+1}(x, y) p_{n+1}^{\prime}\left(x^{\prime}, y^{\prime}\right) \\
& \geq c_{6}\left(p_{n}(x, y)+p_{n+1}(x, y)\right) p_{n}^{\prime}\left(x^{\prime}, y^{\prime}\right) \\
& \geq c_{7} \widetilde{V}\left(\left(x, x^{\prime}\right), n^{1 / \beta}\right)^{-1} \exp \left(-c_{8}\left(\frac{\widetilde{d}\left(\left(x, x^{\prime}\right),\left(y, y^{\prime}\right)\right)^{\beta}}{n}\right)^{1 /(\beta-1)}\right) .
\end{aligned}
$$

Thus $(\widetilde{G}, \widetilde{E}, \widetilde{a})$ satisfies (1.12) and (1.13), and so by Theorem 1.2, it satisfies $\operatorname{PHI}(\beta)$.

Therefore by Corollary $4.11 \widetilde{G}_{C}$ satisfies $\operatorname{CS}(\beta)$ for some $\theta \in(0,1)$. Hence by Proposition 3.4, $\widetilde{G}$ also satisfies $\operatorname{CS}(\beta)$. We now show that this implies that $G$ satisfies $\operatorname{CS}(\beta)$.

Fix a point $0^{\prime} \in G^{\prime}$. Let $R \geq c_{9}$, and $x_{0} \in G$. Using Remark 3.2 we can find a cut-off function $\widetilde{\varphi}$ which is Hölder continuous of order $\theta$ and such that $\varphi=0$ outside $\left(B\left(x_{0}, R\right) \times B^{\prime}\left(0^{\prime}, R\right)\right)^{c}$ and $\widetilde{\varphi}=1$ in $B\left(x_{0}, R / 2\right) \times B^{\prime}\left(0^{\prime}, R / 2\right)$. Let $A^{\prime}=B^{\prime}\left(0^{\prime}, R / 3\right)$, and let

$$
\varphi(x)=\mu_{0}^{\prime}\left(A^{\prime}\right)^{-1} \sum_{x^{\prime} \in A^{\prime}} \widetilde{\varphi}\left(x, x^{\prime}\right) \mu_{0}^{\prime}\left(x^{\prime}\right) .
$$


It is clear that $\varphi$ satisfies conditions (a)-(c) for a cut-off function for $B\left(x_{0}, R\right)$. To verify (d), let $c_{10} \leq s \leq R / 3, I=B(x, s)$ for some $x \in G$, and $f: I^{*} \rightarrow \mathbb{R}$. Extend $f$ to $\widetilde{f}$ on $I^{*} \times G^{\prime}$ by setting $\widetilde{f}\left(x, x^{\prime}\right)=f(x)$. Using Lemma 2.7 we can cover $A^{\prime}$ by balls $J_{i}=B^{\prime}\left(x_{i}^{\prime}, s\right), i=1, \ldots, n$, so that no point is in more than $M$ of the sets $J_{i}^{*}$. We have

$$
\begin{aligned}
& \sum_{x \in I} f(x)^{2} \sum_{y \sim x} a_{x y}|\varphi(x)-\varphi(y)|^{2} \\
& \quad \leq \mu_{0}^{\prime}\left(A^{\prime}\right)^{-1} \sum_{x \in I} \sum_{y \sim x} \sum_{x^{\prime} \in A^{\prime}} a_{x y} f(x)^{2}\left|\widetilde{\varphi}\left(x, x^{\prime}\right)-\widetilde{\varphi}\left(y, x^{\prime}\right)\right|^{2} \mu_{0}^{\prime}\left(x^{\prime}\right) \\
& \quad \leq \mu_{0}^{\prime}\left(A^{\prime}\right)^{-1} \sum_{x \in I} \sum_{y \sim x} \sum_{x^{\prime} \in A^{\prime}} \sum_{y^{\prime} \sim x^{\prime}} a_{x y} a_{x^{\prime} y^{\prime}}^{\prime} \widetilde{f}\left(x, x^{\prime}\right)^{2}\left|\widetilde{\varphi}\left(x, x^{\prime}\right)-\widetilde{\varphi}\left(y, y^{\prime}\right)\right|^{2} \mu_{0}^{\prime}\left(x^{\prime}\right) \\
& \quad \leq \mu_{0}^{\prime}\left(A^{\prime}\right)^{-1} \sum_{i=1}^{n} \sum_{\left(x, x^{\prime}\right) \in I \times J_{i}} \sum_{\left(y, y^{\prime}\right) \sim\left(x, x^{\prime}\right)} a_{x y} a_{x^{\prime} y^{\prime}}^{\prime} \widetilde{f}\left(x, x^{\prime}\right)^{2}\left|\widetilde{\varphi}\left(x, x^{\prime}\right)-\widetilde{\varphi}\left(y, y^{\prime}\right)\right|^{2} \mu_{0}^{\prime}\left(x^{\prime}\right) .
\end{aligned}
$$

Since $V^{\prime}(x, r) \approx r^{\alpha_{1}}$ and $a_{x y}^{\prime}$ is 0 or 1 , there exists $c_{11}$ such that $\mu_{0}^{\prime}\left(x^{\prime}\right) \leq c_{11}$ for all $x^{\prime}$. For each $i$ we use the weighted Sobolev inequality for $\widetilde{\varphi}$ (where we use Remark 3.2 to replace balls in $\widetilde{G}$ by products of balls):

$$
\begin{aligned}
\sum_{\left(x, x^{\prime}\right) \in I \times J_{i}} & \sum_{\left(y, y^{\prime}\right) \sim\left(x, x^{\prime}\right)} a_{x y} a_{x^{\prime} y^{\prime}}^{\prime} \widetilde{f}\left(x, x^{\prime}\right)^{2}\left|\widetilde{\varphi}\left(x, x^{\prime}\right)-\varphi\left(y, y^{\prime}\right)\right|^{2} \\
\leq c_{12}(s / R)^{2 \theta} & \sum_{\left(x, x^{\prime}\right) \in I^{*} \times J_{i}^{*}} \sum_{\left(y, y^{\prime}\right) \sim\left(x, x^{\prime}\right)} a_{x y} a_{x^{\prime} y^{\prime}}^{\prime}\left|\widetilde{f}\left(x, x^{\prime}\right)-\widetilde{f}\left(y, y^{\prime}\right)\right|^{2} \\
& \left.\quad+s^{-\beta} \sum_{\left(x, x^{\prime}\right) \in I^{*} \times J_{i}^{*}} \tilde{f}\left(x, x^{\prime}\right)^{2} \mu_{0}(x) \mu_{0}^{\prime}\left(x^{\prime}\right)\right) \\
= & c_{12}(s / R)^{2 \theta} \mu_{0}^{\prime}\left(A^{\prime}\right)\left(\sum_{x \in I^{*}} \sum_{y \sim x} a_{x y}|f(x)-f(y)|^{2}+s^{-\beta} \sum_{x \in I^{*}} f(x)^{2} \mu_{0}(x)\right) .
\end{aligned}
$$

Summing over $i$ we obtain

$$
\begin{aligned}
\sum_{x \in I} f(x)^{2} & \sum_{y \sim x} a_{x y}|\varphi(x)-\varphi(y)|^{2} \\
& \leq c_{13} M(s / R)^{2 \theta}\left(\sum_{x \in I^{*}} \sum_{y \sim x} a_{x y}|f(x)-f(y)|^{2}+s^{-\beta} \sum_{x \in I^{*}} f(x)^{2} \mu_{0}(x)\right),
\end{aligned}
$$

which is the weighted Sobolev inequality for $\varphi$.

\section{Sobolev inequalities and Elliptic Harnack inequality}

In this section we will prove that the conditions (VD), $P I(\beta)$ and $\operatorname{CS}(\beta)$ imply the elliptic Harnack inequality. We begin with the connection between these conditions and the effective resistance of annuli.

Lemma 5.1. Let $G_{C}$ be the cable system of the graph $G$ which satisfies $\left(p_{0}\right)$ and $(V D)$.

(a) If $P I(\beta)$ holds, then for any $x_{0} \in G_{C}, R \geq 1$,

$$
R_{\mathrm{eff}}\left(B\left(x_{0}, R\right), B\left(x_{0}, 2 R\right)^{c}\right) \leq c_{1} \frac{R^{\beta}}{V\left(x_{0}, R\right)} .
$$


(b) If $C S(\beta)$ holds, then for any $x_{0} \in G_{C}, R \geq 1$,

$$
R_{\mathrm{eff}}\left(B\left(x_{0}, R\right), B\left(x_{0}, 2 R\right)^{c}\right) \geq c_{2} \frac{R^{\beta}}{V\left(x_{0}, 2 R\right)} .
$$

(c) If $G_{C}$ satisfies $C S\left(\beta_{1}\right)$ and $P I\left(\beta_{2}\right)$, then $\beta_{1} \leq \beta_{2}$.

Proof. (a) Let $f$ be the function which attains the minimum on the right hand side of (1.10) when $A=B\left(x_{0}, R\right)$ and $B=B\left(x_{0}, 2 R\right)^{c}$. Let

$$
\bar{f}=\int_{B\left(x_{0}, 3 R\right)} f d \mu / \mu\left(B\left(x_{0}, 3 R\right)\right) .
$$

Choose $y_{0}$ so that $d\left(x_{0}, y_{0}\right)=5 R / 2$. Then by $(2.1)$ we have $V\left(y_{0}, R / 2\right) \geq c_{3} V\left(x_{0}, R\right)$. Depending on whether $\bar{f} \geq 1 / 2$ or $\bar{f}<1 / 2,|f-\bar{f}| \geq 1 / 2$ on either $B\left(x_{0}, R\right)$ or $B\left(y_{0}, R / 2\right)$, and then using $P I(\beta)$ we have

$$
\begin{aligned}
V\left(x_{0}, R\right) & \leq c_{4} \int_{B\left(x_{0}, 3 R\right)}(f-\bar{f})^{2} d \mu \leq c_{5} R^{\beta} \int_{B\left(x_{0}, 3 R\right)}|\nabla f|^{2} d \mu \\
& =c_{5} R^{\beta} R_{\mathrm{eff}}\left(B\left(x_{0}, R\right), B\left(x_{0}, 2 R\right)^{c}\right)^{-1} .
\end{aligned}
$$

(b) Let $\varphi$ be a cut-off function for $B\left(x_{0}, R\right)$ given by $\operatorname{CS}(\beta)$. Then taking $f \equiv 1$ and $I=B\left(x_{0}, R\right)$ in (3.1) we obtain

$$
R_{\mathrm{eff}}\left(B\left(x_{0}, R / 2\right), B\left(x_{0}, R\right)^{c}\right)^{-1} \leq \int_{I}|\nabla \varphi|^{2} d \mu \leq c_{6} R^{-\beta} \int_{I^{*}} f^{2} d \mu \leq c_{7} \frac{V\left(x_{0}, R\right)}{R^{\beta}} .
$$

(c) is immediate from (a) and (b).

Let us now assume $G_{C}$ satisfies (VD), $P I(\beta)$ and $\operatorname{CS}(\beta)$. Using $\operatorname{CS}(\beta)$ we will obtain weighted Sobolev and Poincaré inequalities which we can then use to drive the Moser iteration.

Fix $x_{0} \in G_{C}$, and let $R \geq 1$. Let $\varphi$ be a cut-off function given by $\operatorname{CS}(\beta)$. Write

$$
\gamma=1+R^{\beta}|\nabla \varphi|^{2} .
$$

Proposition 5.2. Let $I=B(x, s)$ with $s \leq R$. Suppose $f$ and its gradient are square integrable over $I^{*}$. Let $f_{A}=\mu(A)^{-1} \int_{A} f$.

(a) We have

$$
\int_{I} f^{2} \gamma \leq c_{1}(s / R)^{2 \theta} R^{\beta}\left(\int_{I^{*}}|\nabla f|^{2}+\psi(s)^{-1} \int_{I^{*}} f^{2}\right) .
$$

(b) We have

$$
\int_{I}\left(f-f_{I^{*}}\right)^{2} \gamma \leq c_{2}(s / R)^{2 \theta} R^{\beta} \int_{I^{*}}|\nabla f|^{2} .
$$

(c) If $J \subset I$, then

$$
\int_{J} f^{2} \gamma \leq c_{3}\left(R^{\beta}(s / R)^{2 \theta}\right) \int_{I^{*}}|\nabla f|^{2}+\mu(J)^{-1}\left(\int_{J}|f| \gamma\right)^{2} .
$$

(d) We have

$$
\int_{B\left(x_{0}, R\right)} \gamma \leq c_{4} V\left(x_{0}, R\right)
$$


Proof. (a) Using the definition of $\gamma$ and (3.1),

$$
\begin{aligned}
\int_{I} f^{2} \gamma & =\int_{I} f^{2}+R^{\beta} \int_{I} f^{2}|\nabla \varphi|^{2} \\
& \leq \int_{I} f^{2}+c_{5}(s / R)^{2 \theta} R^{\beta} \int_{I^{*}}|\nabla f|^{2}+c_{5}(s / R)^{2 \theta} R^{\beta} \psi(s)^{-1} \int_{I^{*}} f^{2} .
\end{aligned}
$$

Since $\beta \geq 2 \geq 2 \theta$ and $s \leq R$, this implies (a).

For (b), applying (5.1) to $f-f_{I^{*}}$ we have

$$
\int_{I}\left(f-f_{I^{*}}\right)^{2} \gamma \leq c_{6}(s / R)^{2 \theta} R^{\beta}\left(\int_{I^{*}}|\nabla f|^{2}+\psi(s)^{-1} \int_{I^{*}}\left(f-f_{I^{*}}\right)^{2}\right) .
$$

Using $P I(\beta)$ applied to the ball $I^{*}$ we have

$$
\int_{I^{*}}\left(f-f_{I^{*}}\right)^{2} \leq c_{7} \psi(s) \int_{I^{*}}|\nabla f|^{2} .
$$

Substituting (5.4) into (5.3) gives (5.2).

(c) Now let $b=\int_{J} f \gamma / \int_{J} \gamma$. Then

$$
\begin{aligned}
\int_{J} f^{2} \gamma & =\int_{J}(f-b)^{2} \gamma+b^{2} \int_{J} \gamma \\
& \leq \int_{J}\left(f-f_{I^{*}}\right)^{2} \gamma+\left(\int_{J} \gamma\right)^{-1}\left(\int_{J} f \gamma\right)^{2} . \\
& \leq \int_{I}\left(f-f_{I^{*}}\right)^{2} \gamma+\mu(J)^{-1}\left(\int_{J}|f| \gamma\right)^{2} .
\end{aligned}
$$

Using (5.2) to bound the first term in (5.5) completes the proof of (c).

(d) follows from (a) by taking $s=R$ and $f=1$ and using (VD).

Our next result is a weighted Nash inequality. For any set $J \subset G_{C}$ set

$$
J^{s}=\{y: d(y, J) \leq s\} .
$$

Proposition 5.3. Let $s \leq R$ and $J \subset B\left(x_{0}, R\right)$ be a finite union of balls of radius s. Suppose the gradient of $f$ is square integrable over $J^{s}$ and $\int_{J^{s}} f^{2} \gamma<\infty$. There exist $c_{1}<\infty$ and $\alpha_{3} \in(0,1)$ such that

$$
\begin{aligned}
& \mu(J)^{-1} \int_{J} f^{2} \gamma \\
& \quad \leq c_{1}\left[R^{\beta} \mu(J)^{-1} \int_{J^{s}}|\nabla f|^{2}+(s / R)^{-2 \theta} \mu(J)^{-1} \int_{J} f^{2} \gamma\right]^{1-\alpha_{3}}\left[\mu(J)^{-1} \int_{J}|f| \gamma\right]^{2 \alpha_{3}} .
\end{aligned}
$$

Proof. Suppose that $0<t<s$. Using Lemma 2.7 we can cover $J$ by balls $B\left(x_{i}, t\right)$ with $x_{i} \in J$ so that any point of $J^{s}$ is in at most $M$ of the balls $B\left(x_{i}, 2 t\right)$. Set $B_{i}=B\left(x_{i}, t\right) \cap J, B_{i}^{*}=B\left(x_{i}, 2 t\right)$. Then $\bigcup_{i} B_{i}=J, \bigcup_{i} B_{i}^{*} \subset J^{s}$, and $\sum \mu\left(B_{i}^{*}\right) \leq$ $M \mu\left(J^{s}\right)$.

As $J$ is a union of balls, for each $i$ there exists $y_{i}$ so that $d\left(x_{i}, y_{i}\right)=t / 2$ and $B\left(y_{i}, t / 2\right) \subset J$. Then by $(2.1)$

$$
\frac{\mu(J)}{\mu\left(B_{i}\right)} \leq \frac{\mu(J)}{\mu\left(B\left(y_{i}, t / 2\right)\right)} \leq c_{2}\left(\frac{R}{t}\right)^{\alpha_{2}} .
$$


By Proposition 5.2(c), and (5.6)

$$
\begin{aligned}
\int_{J} f^{2} \gamma & \leq \sum_{i} \int_{B_{i}} f^{2} \gamma \\
& \leq c_{3}(t / R)^{2 \theta} R^{\beta} \sum_{i} \int_{B_{i}^{*}}|\nabla f|^{2}+\sum_{i} \frac{1}{\mu\left(B_{i}\right)}\left(\int_{B_{i}}|f| \gamma\right)^{2} \\
& \leq c_{4}(t / R)^{2 \theta} R^{\beta} M \int_{J^{*}}|\nabla f|^{2}+c_{5}(R / t)^{\alpha_{2}} \mu(J)^{-1}\left(\sum_{i} \int_{B_{i}}|f| \gamma\right)^{2} \\
& \leq c_{6}(t / R)^{2 \theta} R^{\beta} \int_{J^{*}}|\nabla f|^{2}+c_{7}(R / t)^{\alpha_{2}} \mu(J)^{-1}\left(\int_{J}|f| \gamma\right)^{2} .
\end{aligned}
$$

Hence

$$
\mu(J)^{-1} \int_{J} f^{2} \gamma \leq c_{8}\left[(t / R)^{2 \theta} A+(R / t)^{\alpha_{2}} B\right]
$$

where

$$
A=\left[R^{\beta} \mu(J)^{-1} \int_{J^{*}}|\nabla f|^{2}+(s / R)^{-2 \theta} \mu(J)^{-1} \int_{J} f^{2} \gamma\right], \quad B=\left[\mu(J)^{-1} \int_{J}|f| \gamma\right]^{2} .
$$

If $t \geq s,(5.7)$ is obvious.

We choose $t$ so that the two terms on the right hand side of (5.7) are equal. Thus $(t / R)^{2 \theta+\alpha_{2}}=B / A$, and substituting this into (5.7) completes the proof, with $\alpha_{3}=2 \theta /\left(2 \theta+\alpha_{2}\right)$. Note that if $\theta=1$ and $\alpha_{2}=d$, we obtain the powers in the standard Nash inequality.

We now use this to derive a weighted Sobolev inequality.

Theorem 5.4. Let $s, J$, and $f$ be as in Proposition 5.3. There exist $\kappa>1$ and $c_{1}<\infty$ such that

$$
\left(\mu(J)^{-1} \int_{J}|f|^{2 \kappa} \gamma\right)^{1 / \kappa} \leq c_{1}\left[R^{\beta} \mu(J)^{-1} \int_{J^{s}}|\nabla f|^{2}+(s / R)^{-2 \theta} \mu(J)^{-1} \int_{J} f^{2} \gamma\right] .
$$

Proof. The proof is similar to the one in [BB3], but we correct some algebraic errors in the proof there. Since $\left|\nabla\left(f^{+}\right)\right| \leq|\nabla f|$ a.e. and $|f| \leq f^{+}+f^{-}$, it suffices to consider nonnegative $f$. Write

$$
\begin{aligned}
& A_{0}(f)=\mu(J)^{-1} R^{\beta} \int_{J^{s}}|\nabla f|^{2}+(s / R)^{-2 \theta} \mu(J)^{-1} \int_{J} f^{2} \gamma, \\
& B_{0}(f)=\left(\mu(J)^{-1} \int_{J}|f| \gamma\right)^{2} .
\end{aligned}
$$

Multiplying $f$ by $A_{0}(f)^{-1 / 2}$, it is enough to prove

$$
\int_{J}|f|^{2 \kappa} \gamma \leq c_{1} \mu(J) \quad \text { if } A_{0}(f)=1 .
$$

Set

$$
p_{n}=\mu(J)^{-1} \int_{\left\{f \geq 2^{n}\right\} \cap J} \gamma .
$$

Let $f_{n}=\left(f \wedge 2^{n+1}\right)-\left(f \wedge 2^{n}\right)$; note that $f_{n} \leq 2^{n}$, that $f_{n}=2^{n}$ on $J \cap\left\{f \geq 2^{n+1}\right\}$, and that $f_{n}=0$ on $\left\{f<2^{n}\right\}$. Therefore

$$
\mu(J)^{-1} \int_{J} f_{n} \gamma=\mu(J)^{-1} \int_{\left\{f \geq 2^{n}\right\} \cap J} f_{n} \gamma \leq \mu(J)^{-1} \int_{\left\{f \geq 2^{n}\right\} \cap J} 2^{n} \gamma=2^{n} p_{n}
$$


while

$$
\mu(J)^{-1} \int_{J} f_{n}^{2} \gamma \geq \mu(J)^{-1} \int_{\left\{f \geq 2^{n+1}\right\} \cap J} f_{n}^{2} \gamma=2^{2 n} p_{n+1} .
$$

Since $\int_{J} f_{n}^{2} \gamma \leq \int_{J} f^{2} \gamma$ and $\int_{J^{*}}\left|\nabla f_{n}\right|^{2} \leq \int_{J^{*}}|\nabla f|^{2}$, we have $A_{0}\left(f_{n}\right) \leq A_{0}(f)$. As $s \leq R$, from (5.11) we deduce

$$
p_{n} \leq c_{2} 2^{-2(n-1)}(s / R)^{-2 \theta} \mu(J)^{-1} \int_{J} f_{n-1}^{2} \gamma \leq c_{3} 2^{-2 n} A_{0}\left(f_{n-1}\right) \leq c_{4} 2^{-2 n} .
$$

Applying Proposition 5.3 to $f_{n}$ we have, using the fact that $A_{0}(f) \leq 1$ and $s \leq R$,

$$
\mu(J)^{-1} \int_{J} f_{n}^{2} \gamma \leq c_{5} B_{0}\left(f_{n}\right)^{\alpha_{3}} .
$$

Using this, (5.10), and (5.11) we obtain

$$
2^{2 n} p_{n+1} \leq c_{6} \mu(J)^{-1} \int_{J} f_{n}^{2} \gamma \leq c_{7}\left(\mu(J)^{-1} \int_{J} f_{n} \gamma\right)^{2 \alpha_{3}} \leq c_{8}\left(2^{n} p_{n}\right)^{2 \alpha_{3}} .
$$

By (5.12) $2^{2 n} p_{n}$ is bounded. Using (5.12), (5.13), and the fact that $\alpha_{3}<1$,

$$
2^{2 n} p_{n+1} \leq c_{8}\left(2^{n} p_{n}\right)^{2 \alpha_{3}} \leq c_{9} 2^{-2 \alpha_{3} n} .
$$

Hence

$$
p_{n} \leq c_{10} 2^{-n\left(2+2 \alpha_{3}\right)} .
$$

Since

$$
\mu(J)^{-1} \int_{J}|f|^{2 \kappa} \gamma \leq c_{11} \sum_{n=0}^{\infty} 2^{2 n \kappa} p_{n}
$$

we deduce (5.9) if $\kappa \in\left(1,1+\alpha_{3}\right)$.

The following result is proved exactly as in Moser [M1, Lemma 4.

Proposition 5.5. Let $D \subset G_{C}$ be a domain in $G_{C}$, and suppose $u$ is $\mathcal{L}$-harmonic in $D, v=u^{k}$, where $k \in \mathbb{R}, k \neq 1 / 2$, and $\eta$ is supported in $D$. Suppose the gradient of $\eta$ is square integrable over $D$. Then

$$
\int_{D} \eta^{2}|\nabla v|^{2} \leq c_{1}\left(\frac{2 k}{2 k-1}\right)^{2} \int_{D}|\nabla \eta|^{2} v^{2}
$$

Corollary 5.6. Let $D, u, k, v$ be as above. Suppose $\eta>0$ on $D, \eta=0$ on $\partial D, \eta$ is continuous on $\bar{D}$, and the gradient of $\eta$ is square integrable over $D$. Then

$$
\int_{D} \eta^{2}|\nabla v|^{2} d \mu \leq c_{1}\left(\frac{2 k}{2 k-1}\right)^{2} \int_{D}|\nabla \eta|^{2} v^{2} d \mu .
$$

Proof. Since $\eta$ is continuous on $\bar{D}, 0$ on $\partial D$ and positive in $D$, then $\eta_{\varepsilon}=(\eta-\varepsilon)^{+}$ will be 0 in a neighborhood of $\partial D$. Applying Proposition 5.5 we obtain

$$
\int_{D} \eta_{\varepsilon}^{2}|\nabla v|^{2} \leq c_{2}\left(\frac{2 k}{2 k-1}\right)^{2} \int_{D}\left|\nabla \eta_{\varepsilon}\right|^{2} v^{2} \leq c_{2}\left(\frac{2 k}{2 k-1}\right)^{2} \int_{D}|\nabla \eta|^{2} v^{2}
$$

Now let $\varepsilon \rightarrow 0$.

Let $u$ be $\mathcal{L}$-harmonic and nonnegative in $B\left(x_{0}, 4 R\right)$. By looking at $u+\varepsilon$ and letting $\varepsilon \downarrow 0$ we may without loss of generality suppose $u$ is strictly positive. The usual Harnack inequality in $\mathbb{R}$, combined with the local Harnack inequality for graphs, implies that $u$ and $u^{-1}$ are continuous and bounded (by a constant depending on $u, x, r$, and $R$ ) on any ball $\overline{B(x, r)}$ in $B\left(x_{0}, 4 R\right)$. By Proposition 5.5, the gradient 
of powers of $u$ will be square integrable over bounded subsets of $G_{C}$. We take $\varphi$ to be a cut-off function for $B\left(x_{0}, R\right)$.

We will need the following estimate.

Proposition 5.7. Let $w=\log u$. There exists $c_{1}$ such that

$$
\int_{B\left(x_{0}, 2 R\right)}|\nabla w|^{2} \leq c_{1} \frac{V\left(x_{0}, R\right)}{R^{\beta}} .
$$

Proof. Again, this is essentially Moser's proof. Let $\varphi_{1}(x)$ be a cut-off function given by $\operatorname{CS}(\beta)$ for the ball $B\left(x_{0}, 4 R\right)$. So

$$
\int_{B\left(x_{0}, 2 R\right)}|\nabla w|^{2} \leq c_{2} \int \varphi_{1}^{2}|\nabla w|^{2}
$$

By Lemma 2.3

$$
\begin{aligned}
0 & =\int \frac{\varphi_{1}^{2}}{u} \mathcal{L} u d \mu=-\int \nabla\left(\varphi_{1}^{2} / u\right) \cdot \nabla u d \mu \\
& =-\int\left(2 \frac{\varphi_{1}}{u} \nabla \varphi_{1} \cdot \nabla u-\frac{\varphi_{1}^{2}}{u^{2}}|\nabla u|^{2}\right) d \mu \\
& =-2 \int \varphi_{1} \nabla \varphi_{1} \cdot \nabla w d \mu+\int \varphi_{1}^{2}|\nabla w|^{2} d \mu .
\end{aligned}
$$

So

$$
\int \varphi_{1}^{2}|\nabla w|^{2} d \mu \leq c_{3}\left|\int \varphi_{1} \nabla \varphi_{1} \cdot \nabla w d \mu\right| \leq c_{3}\left(\int\left|\nabla \varphi_{1}\right|^{2} d \mu\right)^{1 / 2}\left(\int \varphi_{1}^{2}|\nabla w|^{2} d \mu\right)^{1 / 2} .
$$

Dividing and squaring,

$$
\int \varphi_{1}^{2}|\nabla w|^{2} d \mu \leq c_{4} \int\left|\nabla \varphi_{1}\right|^{2} d \mu .
$$

Finally, using $\operatorname{CS}(\beta)$ in $B\left(x_{0}, 4 R\right)$ with $f=1$ and (VD) we deduce that

$$
\int_{B\left(x_{0}, 4 R\right)}\left|\nabla \varphi_{1}\right|^{2} d \mu \leq c_{5} R^{-\beta} V\left(x_{0}, R\right) .
$$

Proposition 5.8. Let $v$ be either $u$ or $u^{-1}$. There exists $c_{1}$ such that if $B(x, 2 r) \subset$ $B\left(x_{0}, 4 R\right)$ and $0<q<2$, then

$$
\sup _{B(x, r / 2)} v^{2 q} \leq c_{1} V(x, 2 r)^{-1} \int_{B(x, 2 r)}\left(r^{\beta}\left|\nabla v^{q}\right|^{2}+v^{2 q}\right) d \mu .
$$

Proof. If $r<1$ this follows easily from the local Harnack inequality and the linearity of $u$ on each cable, so suppose $r \geq 1$. Let $\varphi_{0}$ be a (regularized) cut-off function given by $\operatorname{CS}(\beta)$ for $B(x, r)$. Let $h_{n}=1-2^{-n}, 0 \leq n \leq \infty$, so that $0=h_{0}<h_{\infty}=1$. For $k \geq 0$ set

$$
\begin{aligned}
\varphi_{k}(x) & =\left(\varphi_{0}(x)-h_{k}\right)^{+}, \\
\gamma_{0}(x) & =1+r^{\beta}\left|\nabla \varphi_{0}(x)\right|^{2} .
\end{aligned}
$$

Set $A_{k}=\left\{x: \varphi_{0}(x)>h_{k}\right\}$, and note that $B(x, r / 2) \subset A_{n_{0}} \subset A_{0} \subset B(x, r)$ for every $n_{0}$. We therefore have, writing $V$ for $V(x, r)$,

$$
c_{2} V \leq \mu\left(A_{k}\right) \leq V, \quad k \geq 0 .
$$


The Hölder condition on $\varphi_{0}$ given by Definition 3.1(c) implies that if $x \in A_{k+1}$ and $y \in A_{k}^{c}$, then $d(x, y) \geq c_{3} r 2^{-k / \theta}$. Set $s_{k}=\frac{1}{2} c_{3} r 2^{-k / \theta}$, and note that $\varphi_{k}>c_{4} 2^{-k}$ on $A_{k+1}^{s_{k}}$. Let $\left\{B_{i}\right\}$ be a cover of $A_{k+1}$ by balls of radius $s_{k} / 2$, and let $J_{k+1}=\bigcup B_{i}$. Write $J_{k+1}^{\prime}=J_{k+1}^{s_{k} / 2}$ and note that $A_{k+1} \subset J_{k+1} \subset J_{k+1}^{\prime} \subset A_{k+1}^{s_{k}}$.

From Theorem 5.4 with $f=v^{p}$ and $s$ replaced by $s_{k} / 2$,

$$
\begin{aligned}
\left(V^{-1} \int_{A_{k+1}} f^{2 \kappa} \gamma_{0} d \mu\right)^{1 / \kappa} & \leq\left(V^{-1} \int_{J_{k+1}} f^{2 \kappa} \gamma_{0} d \mu\right)^{1 / \kappa} \\
& \leq c_{5} V^{-1}\left[r^{\beta} \int_{J_{k+1}^{\prime}}|\nabla f|^{2} d \mu+\left(r / s_{k}\right)^{2 \theta} \int_{J_{k+1}^{\prime}} f^{2} \gamma_{0} d \mu\right] \\
& \leq c_{6} V^{-1}\left[r^{\beta} \int_{A_{k+1}^{s_{k}}}|\nabla f|^{2} d \mu+2^{2 k} \int_{A_{k}} f^{2} \gamma_{0} d \mu\right] .
\end{aligned}
$$

We now control the first term in (5.14) using Corollary 5.6.

$$
\begin{aligned}
r^{\beta} \int_{A_{k+1}^{s_{k}}}|\nabla f|^{2} & \leq r^{\beta}\left(c_{7} 2^{-k}\right)^{-2} \int_{A_{k+1}^{s_{k}}} \varphi_{k}^{2}|\nabla f|^{2} \\
& \leq c_{8} 2^{2 k} r^{\beta} \int_{A_{k}} \varphi_{k}^{2}|\nabla f|^{2} \\
& \leq c_{9} 2^{2 k} r^{\beta}\left(\frac{2 p}{2 p-1}\right)^{2} \int_{A_{k}} f^{2}\left|\nabla \varphi_{k}\right|^{2} \\
& \leq c_{10} 2^{2 k}\left(\frac{2 p}{2 p-1}\right)^{2} \int_{A_{k}} f^{2} \gamma_{0} .
\end{aligned}
$$

We therefore deduce that

$$
\left(V^{-1} \int_{A_{k+1}} f^{2 \kappa} \gamma_{0}\right)^{1 / \kappa} \leq c_{11}\left(\frac{2 p}{2 p-1}\right)^{2} 2^{2 k} V^{-1} \int_{A_{k}} f^{2} \gamma_{0}
$$

Choose $q^{\prime}>0$ such that $\inf _{m \in \mathbb{Z}}\left|q^{\prime} \kappa^{m}-\frac{1}{2}\right| \geq c_{12}>0$. Suppose first that $q_{0}=q^{\prime} \kappa^{-i}$ for some $i$. Let $p_{n}=2 q_{0} \kappa^{n}$ for $n \geq 0$, and write

$$
\Psi_{k}=\left[\mu\left(A_{k}\right)^{-1} \int_{A_{k}} v^{p_{k}} \gamma_{0}\right]^{1 / p_{k}} .
$$

Note that $p_{k+1} / 2 \kappa=p_{k} / 2$. Applying (5.15) to $f=v^{p_{k+1} /(2 \kappa)}=v^{p_{k} / 2}$ we have

or

$$
\begin{aligned}
\Psi_{k+1}^{p_{k+1} / \kappa} & =\left(\mu\left(A_{k+1}\right)^{-1} \int_{A_{k+1}} v^{p_{k+1}} \gamma_{0}\right)^{1 / \kappa} \\
& \leq c_{13} 2^{2 k}\left(\mu\left(A_{k}\right)^{-1} \int_{A_{k}} v^{p_{k}} \gamma_{0}\right)=c_{13} 2^{2 k} \Psi_{k}^{p_{k}},
\end{aligned}
$$

Hence for every $m$

$$
\Psi_{k+1} \leq\left(c_{13} 2^{2 k}\right)^{1 / p_{k}} \Psi_{k}
$$

$$
\log \Psi_{m} \leq \log \Psi_{0}+\sum_{k=1}^{m} p_{k}^{-1} \log \left(c_{13} 2^{2 k}\right) .
$$

As the sum in (5.16) converges and $\sup _{B(x, r / 2)} v \leq \lim \sup _{m \rightarrow \infty} \Psi_{m}$, we have

$$
\sup _{B(x, r / 2)} v \leq c_{14} \Psi_{0} \leq c_{15}\left(V^{-1} \int_{B(x, r)} v^{2 q_{0}} \gamma_{0}\right)^{1 /\left(2 q_{0}\right)} .
$$


Now let $q \in(0,2)$. We can take $q_{0}=q^{\prime} \kappa^{-i}<q$. Then by Hölder's inequality and Proposition 5.2(d)

$$
\begin{aligned}
V^{-1} \int_{B(x, r)} v^{2 q_{0}} \gamma_{0} & \leq\left(V^{-1} \int_{B(x, r)} v^{2 q} \gamma_{0}\right)^{q_{0} / q}\left(V^{-1} \int_{B(x, r)} \gamma_{0}\right)^{1-q_{0} / q} \\
& \leq c_{16}\left(V^{-1} \int_{B(x, r)} v^{2 q} \gamma_{0}\right)^{q_{0} / q}
\end{aligned}
$$

Thus

$$
\sup _{B(x, r / 2)} v^{2 q} \leq c_{17} V^{-1} \int_{B(x, r)} v^{2 q} \gamma_{0}
$$

By Proposition 5.2(a) and (VD) this implies

$$
\sup _{B(x, r / 2)} v^{2 q} \leq c_{18} V(x, 2 r)^{-1} \int_{B(x, 2 r)}\left(r^{\beta}\left|\nabla v^{q}\right|^{2}+v^{2 q}\right) .
$$

We now follow the ideas of Moser [M3] to link the $L^{\infty}$ norms of $u$ and $u^{-1}$. Recall that $\varphi$ is a cut-off function for $B\left(x_{0}, R\right)$, and let

$$
\gamma=1+R^{\beta}\left|\nabla \varphi^{2}\right|
$$

We define

$$
Q(t)=\{x: \varphi(x)>t\}, \quad 0<t<1,
$$

and write $Q(1)$ for the interior of $\{x: \varphi(x) \geq 1\}$.

Corollary 5.9. Let $1>s>t>0$. There exists $\zeta_{1}>2$ such that if $0<q<\frac{1}{3}$,

$$
\sup _{Q(s)} v^{2 q} \leq c_{1}(s-t)^{-\zeta_{1}} V\left(x_{0}, R\right)^{-1} \int_{Q(t)} v^{2 q} \gamma
$$

Proof. By the maximum principle the supremum of $v^{2 q}$ in $\overline{Q(s)}$ is attained at a point $x^{\prime} \in \partial Q(s)$. Let $\eta=\frac{1}{4}(s-t), s^{\prime}=s-2 \eta$. By the Hölder continuity of $\varphi$ the sets $Q(s)$ and $Q\left(s^{\prime}\right)^{c}$ are separated by a distance of at least $\xi=c_{2} R(s-t)^{1 / \theta}$, so that $B\left(x^{\prime}, \xi\right) \subset Q\left(s^{\prime}\right)$. By Proposition 5.8,

$$
\sup _{B\left(x^{\prime}, \xi / 4\right)} v^{2 q} \leq c_{3} \xi^{\beta} V\left(x^{\prime}, \xi\right)^{-1} \int_{B\left(x^{\prime}, \xi\right)}\left|\nabla v^{q}\right|^{2}+c_{3} V\left(x^{\prime}, \xi\right)^{-1} \int_{B\left(x^{\prime}, \xi\right)} v^{2 q} .
$$

Note that by (2.1) we have

$$
\frac{V\left(x_{0}, R\right)}{V\left(x^{\prime}, \xi\right)} \leq c_{4}\left(\frac{d\left(x^{\prime}, x_{0}\right)+R}{\xi}\right)^{\alpha_{2}} \leq c_{5}(s-t)^{-\alpha_{2} / \theta}
$$

Using (5.19),

$$
\sup _{Q(s)} v^{2 q} \leq c_{6} \xi^{\beta} V\left(x^{\prime}, \xi\right)^{-1} \int_{Q\left(s^{\prime}\right)}\left|\nabla v^{q}\right|^{2}+c_{6} V\left(x^{\prime}, \xi\right)^{-1} \int_{Q\left(s^{\prime}\right)} v^{2 q} .
$$

Let

$$
\varphi_{s t}=(s \wedge \varphi-t)^{+}
$$


and observe that $\left|\nabla \varphi_{s t}\right| \leq|\nabla \varphi|$. Now $\varphi_{s t} \geq c_{7}(s-t)$ on $Q\left(s^{\prime}\right)$, so we have, using Corollary 5.6,

$$
\begin{aligned}
\int_{Q\left(s^{\prime}\right)}\left|\nabla v^{q}\right|^{2} & \leq c_{7}(s-t)^{-2} \int_{Q\left(s^{\prime}\right)}\left|\nabla v^{q}\right|^{2} \varphi_{s t}^{2} \\
& \leq c_{7}(s-t)^{-2} \int_{Q(t)}\left|\nabla v^{q}\right|^{2} \varphi_{s t}^{2} \\
& \leq c_{8}(s-t)^{-2} \int_{Q(t)}\left|\nabla \varphi_{s t}\right|^{2} v^{2 q} \\
& \leq c_{9}(s-t)^{-2} R^{-\beta} \int_{Q(t)} v^{2 q} \gamma
\end{aligned}
$$

Thus

$$
\begin{aligned}
\sup _{Q(s)} v^{2 q} & \leq c_{10}(\xi / R)^{\beta}(s-t)^{-2} V\left(x^{\prime}, \xi\right)^{-1} \int_{Q(t)} v^{2 q} \gamma+c_{10} V\left(x^{\prime}, \xi\right)^{-1} \int_{Q(t)} v^{2 q} \\
& \leq c_{11} V\left(x^{\prime}, \xi\right)^{-1}(s-t)^{-2} \int_{Q(t)} v^{2 q} \gamma \\
& \leq c_{12} V\left(x_{0}, R\right)^{-1}(s-t)^{-2-\alpha_{2} / \theta} \int_{Q(t)} v^{2 q} \gamma
\end{aligned}
$$

So taking $\zeta_{1}=2+\alpha_{2} / \theta$ we obtain (5.18).

Now let $w=\log u$, and write $\bar{w}=V\left(x_{0}, R\right)^{-1} \int_{B\left(x_{0}, R\right)} w d \mu$.

Corollary 5.10. Let $1 \geq s>t>0$. Then

$$
\int_{\{|w-\bar{w}|>A\} \cap Q(s)} \gamma \leq \frac{c_{1} V\left(x_{0}, R\right)}{A^{2}} .
$$

Proof. By Chebyshev's inequality, Proposition 5.2(b) and Proposition 5.7

$$
\begin{aligned}
A^{2} \int_{\{|w-\bar{w}|>A\} \cap Q(s)} \gamma & \leq \int_{\{|w-\bar{w}|>A\} \cap Q(s)}|w-\bar{w}|^{2} \gamma \\
& \leq \int_{Q(s)}|w-\bar{w}|^{2} \gamma \\
& \leq \int_{B\left(x_{0}, R\right)}|w-\bar{w}|^{2} \gamma \\
& \leq c_{2} R^{\beta} \int_{B\left(x_{0}, 2 R\right)}|\nabla w|^{2} \leq c_{3} V\left(x_{0}, R\right) .
\end{aligned}
$$

Without loss of generality, we multiply $u$ by a constant so that

$$
V\left(x_{0}, R\right)^{-1} \int_{B\left(x_{0}, R\right)} \log v=\bar{w}=0 .
$$

Recall that $v$ is either $u$ or $u^{-1}$ and define $\Phi(t)=\sup _{\bar{Q}(t)} \log v$.

Lemma 5.11. Let $1 \geq s>t \geq \frac{1}{2}$. Then

$$
\Phi(s) \leq \frac{3}{4} \Phi(t)+c_{1}(s-t)^{-\zeta_{1}} .
$$


Proof. Fix $t$ and write $\Phi$ for $\Phi(t)$. Let $c_{2}>e$ satisfy $c_{2}=6 \log c_{2}$. If $\Phi(t) \leq c_{2}$, then

$$
\Phi(s) \leq \Phi(t) \leq \frac{3}{4} \Phi(t)+\frac{1}{4} c_{2},
$$

so that (5.20) holds provided $c_{1} 2^{-\zeta_{1}} \geq c_{2} / 4$.

Now suppose $\Phi>c_{2}$. From Proposition 5.2(d) we have $\int_{Q(t)} \gamma \leq c_{3} V\left(x_{0}, R\right)$. By Corollary 5.10 and the fact that $v^{p} \leq e^{p \Phi}$ on $Q(t)$,

$$
\begin{aligned}
\int_{Q(t)} v^{2 p} \gamma & =\int_{Q(t) \cap\{\log v \geq \Phi / 2\}} v^{2 p} \gamma+\int_{Q(t) \cap\{\log v<\Phi / 2\}} v^{2 p} \gamma \\
& \leq e^{2 p \Phi} \int_{Q(t) \cap\{\log v \geq \Phi / 2\}} \gamma+e^{p \Phi} \int_{Q(t) \cap\{\log v<\Phi / 2\}} \gamma \\
& \leq \frac{4 c_{4} e^{2 p \Phi}}{\Phi^{2}} V\left(x_{0}, R\right)+e^{p \Phi} \int_{Q(t)} \gamma \\
& \leq c_{5}\left(\frac{e^{2 p \Phi}}{\Phi^{2}}+e^{p \Phi}\right) V\left(x_{0}, R\right) .
\end{aligned}
$$

Let $p=\frac{2}{\Phi} \log \Phi$, so that $e^{p \Phi}=\Phi^{2}$. As $\Phi>c_{2}$ we have $p<\left(2 / c_{2}\right) \log c_{2}=\frac{1}{3}$. So

$$
V\left(x_{0}, R\right)^{-1} \int_{Q(t)} v^{2 p} \gamma \leq c_{5} e^{p \Phi}\left(1+\frac{e^{p \Phi}}{\Phi^{2}}\right)=2 c_{5} e^{p \Phi} .
$$

Therefore by Corollary 5.9,

$$
\begin{aligned}
\Phi(s) & =\frac{1}{2 p} \log \left[\sup _{Q(s)} v^{2 p}\right] \\
& \leq \frac{1}{2 p} \log \left[c_{6}(s-t)^{-\zeta_{1}} V\left(x_{0}, R\right)^{-1} \int_{Q(t)} v^{2 p} \gamma\right] \\
& \leq \frac{1}{2 p} \log \left[c_{7}(s-t)^{-\zeta_{1}} e^{p \Phi}\right] . \\
& =\left[1+\frac{\log \left(c_{7}(s-t)^{-\zeta_{1}}\right)}{2 \log \Phi}\right] \frac{\Phi}{2} .
\end{aligned}
$$

Without loss of generality we may take $c_{7}$ larger than $c_{2}$. If $\Phi(t) \geq c_{7}(s-t)^{-\zeta_{1}}$, then by (5.21) $\Phi(s) \leq \frac{3}{4} \Phi(t)$, and (5.20) is satisfied. If, on the other hand, $\Phi(t) \leq$ $c_{7}(s-t)^{-\zeta_{1}}$, then since $\Phi(s) \leq \Phi(t)$, we have (5.20) satisfied with $c_{1}=c_{7}$.

Theorem 5.12. There exists $c_{1}$ such that if $u$ is nonnegative and $\mathcal{L}^{\prime}$-harmonic in $B\left(x_{0}, 4 R\right)$, then

$$
\sup _{B\left(x_{0}, R / 2\right)} u \leq c_{1} \inf _{B\left(x_{0}, R / 2\right)} u
$$

Proof. Since $u$ is linear on each cable and $B\left(x_{0}, 4 R\right) \cap G$ is a finite set, then $u$ is continuous and bounded in $B\left(x_{0}, R\right)$. We need to show we can bound the ratio of the supremum of $u$ to the infimum of $u$ in $B\left(x_{0}, R / 2\right)$ by a constant not depending on $u$. Multiplying $u$ by a constant we can assume $\int_{B\left(x_{0}, R\right)} \log u=0$. First let $v=u$. 
Choose $t_{j}=1 /(j+1)$, so that $t_{0}=1$ and $t_{i} \downarrow 0$. Then by Lemma 5.11,

$$
\begin{aligned}
\Phi\left(t_{0}\right) & \leq \frac{3}{4} \Phi\left(t_{1}\right)+c_{2}\left(t_{0}-t_{1}\right)^{-\zeta_{1}} \\
& \leq\left(\frac{3}{4}\right)^{2} \Phi\left(t_{2}\right)+c_{2}\left(t_{0}-t_{1}\right)^{-\zeta_{1}}+\frac{3}{4} c_{2}\left(t_{1}-t_{2}\right)^{-\zeta_{1}} \\
& \leq \cdots \\
& \leq\left(\frac{3}{4}\right)^{n} \Phi\left(t_{n}\right)+\sum_{i=1}^{n}\left(\frac{3}{4}\right)^{i-1} c_{2}\left(t_{i-1}-t_{i}\right)^{-\zeta_{1}},
\end{aligned}
$$

for any $n \geq 0$. Since $\Phi\left(t_{n}\right) \leq \sup _{B\left(x_{0}, R\right)} \log v<\infty$, and

$$
\sum_{i=1}^{\infty}\left(\frac{3}{4}\right)^{i-1} c_{2}\left(t_{i-1}-t_{i}\right)^{-\zeta_{1}}=c_{3}<\infty
$$

we obtain

$$
\sup _{B\left(x_{0}, R / 2\right)} \log u \leq c_{3} .
$$

Now let $v=u^{-1} ; \log v=-\log u$ so we still have $\int_{B\left(x_{0}, R\right)} \log v=0$. The same argument as above now implies $\sup _{B\left(x_{0}, R / 2\right)} \log v \leq c_{3}$, or

$$
\inf _{B\left(x_{0}, R / 2\right)} \log u \geq-c_{3} .
$$

Combining we deduce

$$
e^{-c_{3}} \leq \inf _{B\left(x_{0}, R / 2\right)} u \leq \sup _{B\left(x_{0}, R / 2\right)} u \leq e^{c_{3}},
$$

which is what we wanted to prove.

Proof of Theorem 1.5. First we show that (a) implies (b). By Propositions 3.3 and $3.5, P I(\beta)$ and $\operatorname{CS}(\beta)$ hold for $G_{C}$. The elliptic Harnack inequality (EHI) for $G_{C}$ then follows from Theorem 5.12 by a covering argument. By Corollary 2.5, (EHI) holds for $G$. Lemma 5.1 shows that $G_{C}$ satisfies $\left(R_{\beta}\right)$, and hence by Proposition 3.6, $G$ does also. Hence $(G, E, a)$ satisfies condition (d) of Theorem 1.2, and (b) follows from that theorem.

Now suppose that (b) holds. Then $(G, E, a)$ satisfies $P I(\beta)$ by Lemma 4.1, and $\operatorname{CS}(\beta)$ by Theorem 4.12. Thus (b) implies (a).

Proof of Theorem 1.1. The conditions $P I(\beta)$ and $\operatorname{CS}(\beta)$ are obviously stable. So the conclusion of Theorem 1.1 follows from Lemma 2.1 and Theorem 1.5.

\section{ACKNOWLEDGMENT}

The authors wish to thank Alexander Grigor'yan for some helpful discussions.

\section{REFERENCES}

[A] D.G. Aronson, Bounds on the fundamental solution of a parabolic equation. Bull. Amer. Math. Soc. 73 (1967), 890-896. MR 36:534

[B1] M.T. Barlow. Which values of the volume growth and escape time exponent are possible for a graph? To appear Rev. Math. Iberoamericana.

[BB1] M.T. Barlow, R.F. Bass, Brownian motion and harmonic analysis on Sierpinski carpets. Canad. J. Math. 51 (1999), 673-744. MR 2000i:60083

[BB2] M.T. Barlow, R.F. Bass. Random walks on graphical Sierpinski carpets. In: Random walks and discrete potential theory, ed. M. Piccardello, W. Woess, Symposia Mathematica XXXIX Cambridge Univ. Press, Cambridge, 1999. MR 2002c:60116 
[BB3] M.T. Barlow, R.F. Bass. Divergence form operators on fractal-like domains. J. Funct. Analysis 175 (2000), 214-247. MR 2001i:58071

[BCG] M. Barlow, T. Coulhon, A. Grigor'yan. Manifolds and graphs with slow heat kernel decay. Invent. Math. 144 (2001), 609-649. MR 2002b:58029

[BPY] M. Barlow, J. Pitman, M. Yor. On Walsh's Brownian motions. Sém. Prob. XXIII, 275293, Lecture Notes in Math., 1372, Springer, Berlin, 1989. MR 91a:60204

[Da] E.B. Davies. Heat kernels and spectral theory. Cambridge University Press, 1989. MR 92a:35035

[D1] T. Delmotte. Parabolic Harnack inequality and estimates of Markov chains on graphs. Rev. Math. Iberoamericana 15 (1999), 181-232. MR 2000b:35103

[D2] T. Delmotte. Graphs between the elliptic and parabolic Harnack inequalities. Potential Anal. 16 (2002), no. 2, 151-168. MR 2003b:39019

[FS] E.B. Fabes and D.W. Stroock, A new proof of Moser's parabolic Harnack inequality via the old ideas of Nash. Arch. Mech. Rat. Anal. 96 (1986), 327-338. MR 88b:35037

[FOT] M. Fukushima, Y. Oshima, and M. Takeda, Dirichlet Forms and Symmetric Markov Processes. de Gruyter, Berlin, 1994. MR 96f:60126

[Gr] A.A. Grigor'yan. The heat equation on noncompact Riemannian manifolds. Math. USSR Sbornik 72 (1992), 47-77. MR 92h:58189

[GT1] A. Grigor'yan, A. Telcs. Sub-Gaussian estimates of heat kernels on infinite graphs. Duke Math. J. 109, (2001), 452-510. MR 2003a:35085

[GT2] A. Grigor'yan, A. Telcs. Harnack inequalities and sub-Gaussian estimates for random walks. Math. Annalen 324 (2002), 521-556.

[HK] B.M. Hambly, T. Kumagai. Heat kernel estimates for symmetric random walks on a class of fractal graphs and stability under rough isometries. To appear Proc. Symp. Pure Math.

[HSC] W. Hebisch, L. Saloff-Coste. On the relation between elliptic and parabolic Harnack inequalities. Ann. Inst. Fourier (Grenoble) 51 (2001), 1437-1481. MR 2002g:58024

[Jo] O.D. Jones. Transition probabilities for the simple random walk on the Sierpinski graph. Stoch. Proc. Appl. 61 (1996), 45-69. MR 97b:60115

$[\mathrm{K}] \quad$ S. Kusuoka: Dirichlet forms on fractals and products of random matrices. Publ. RIMS Kyoto Univ., 25, 659-680 (1989). MR 91m:60142

[M1] J. Moser, On Harnack's inequality for elliptic differential equations. Comm. Pure Appl. Math. 14 (1961), 577-591. MR 28:2356

[M2] J. Moser. On Harnack's inequality for parabolic differential equations. Comm. Pure Appl. Math. 17 (1964), 101-134. MR 28:2357

[M3] J. Moser, On a pointwise estimate for parabolic differential equations. Comm. Pure Appl. Math. 24 (1971), 727-740. MR 44:5603

[N] J. Nash, Continuity of solutions of parabolic and elliptic equations, Amer. Math. J. 80 (1958), 931-954. MR 20:6592

[SC] L. Saloff-Coste, A note on Poincaré, Sobolev, and Harnack inequalities. Inter. Math. Res. Notices (1992), 27-38. MR 93d:58158

[St] K.-T. Sturm. Analysis on local Dirichlet spaces III. The parabolic Harnack inequality. J. Math. Pures. Appl. (9) $\mathbf{7 5}$ (1996), 273-297. MR 97k:31010

[T1] A. Telcs. Local sub-Gaussian transition probability estimates, the strongly recurrent case. Electr. J. Prob. 6 (2001), no. 22, 33 pp. MR 2003a:60066

[T2] A. Telcs. Random walks on graphs with volume and time doubling. Preprint.

[W] J.B. Walsh. A diffusion with a discontinuous local time. Temps Locaux, Astérisque 52-53 (1978), 37-45.

Department of Mathematics, University of British Columbia, Vancouver, Canada V6T $1 \mathrm{Z} 2$

E-mail address: barlow@math.ubc.ca

Department of Mathematics, University of Connecticut, Storrs, Connecticut 06269

E-mail address: bass@math.uconn.edu 